\title{
THE ORIGINS OF X-RAY EMISSION FROM THE HOT SPOTS OF FR II RADIO SOURCES
}

\author{
M. J. HaRdCASTLE \\ Department of Physics, University of Bristol, Tyndall Avenue, Bristol BS8 1TL, UK \\ D. E. HARris \\ Harvard-Smithsonian Center for Astrophysics, 60 Garden Street, Cambridge, MA 02138 \\ AND \\ D. M. Worrall and M. Birkinshaw \\ Department of Physics, University of Bristol, Tyndall Avenue, Bristol BS8 1TL, UK \\ Received 2004 March 31; accepted 2004 May 25
}

\begin{abstract}
We use new and archival Chandra data to investigate the X-ray emission from a large sample of compact hot spots of FR II radio galaxies and quasars from the 3C catalog. We find that only the most luminous hot spots tend to be in good agreement with the predictions of a synchrotron self-Compton model with equipartition magnetic fields. At low hot spot luminosities inverse Compton predictions are routinely exceeded by several orders of magnitude, but this is never seen in more luminous hot spots. We argue that an additional synchrotron component of the X-ray emission is present in low-luminosity hot spots and that the hot spot luminosity controls the ability of a given hot spot to produce synchrotron X-rays, probably by determining the high-energy cutoff of the electron energy spectrum. It remains plausible that all hot spots are close to the equipartition condition.
\end{abstract}

Subject headings: galaxies: active — quasars: general — radiation mechanisms: nonthermal — X-rays: galaxies

\section{INTRODUCTION}

The hot spots of powerful FR II radio sources, as observed in the radio, have long been believed to be the observable consequence of a strong terminal shock at the end of the relativistic jets that feed the radio lobes. This picture has to be modified somewhat in the light of the fact that a large number of lobes contain more than one hot spot when observed at high resolution (Laing 1982; Leahy et al. 1997; Hardcastle et al. 1997). Conventionally the most compact feature, which is universally the one that lies at the end of the jet when the jet termination can be observed, is called the "primary" hot spot, while the others are known as "secondary" hot spots. Since standard shock acceleration and energy loss models are often good fits to the radio-to-optical spectra of both primary and secondary hot spots (e.g., Meisenheimer et al. 1989), it seems likely that in many cases the secondary hot spots are also being powered by bulk kinetic energy from the jet.

For some time it has been clear that it is not possible to explain the X-ray emission from hot spots with a single model. Some objects show hot spot X-ray emission with a spectrum consistent with the predictions of a synchrotron self-Compton (SSC) model, in which the synchrotron-emitting electrons inverse Compton (IC) scatter synchrotron photons into the X-ray band; in these objects there is good agreement between the observed flux density and the predictions of an SSC model with a magnetic field close to the value expected for equipartition of energy between the magnetic field and the radiating electrons, and so their X-ray emission is attributed to the SSC process (e.g., Harris et al. 1994; Hardcastle et al. 2002, hereafter H02). On the other hand, objects such as 3C 390.3 (Harris et al. 1998) and Pictor A (Röser \& Meisenheimer 1987) show $\mathrm{X}$-ray emission that is clearly much stronger than the SSC model would predict if the magnetic field had the equipartition value, together with a spectrum that is steeper than the lowfrequency radio spectrum and hence too steep to be inverse
Compton (Wilson et al. 2001; H02). In some, but not all, of these cases, a simple synchrotron spectrum (by which we mean a single power law or a broken, steepening power law in frequency) is a good fit to the radio, optical, and X-ray data points. In addition, there are several sources (the best example being $3 \mathrm{C} 351$; H02) where the X-ray structure is clearly different from that seen in the radio maps, which is impossible in a simple SSC model with a homogeneous magnetic field and electron distribution. In a synchrotron model for some or all of the X-rays, differences in the spatial structure are to be expected, since the synchrotron loss timescale for X-rayemitting electrons (tens of years in a typical equipartition magnetic field) is orders of magnitude less than that for radio-emitting electrons ( $\gtrsim 10^{5} \mathrm{yr}$ ); in fact, in a non-steady state situation (as expected from numerical simulations; e.g., Tregillis et al. 2001) time-varying differences in both spatial and spectral distributions of the radio- and X-ray-emitting electrons are more or less required by the physics.

Until now, however, it has not been clear why some hot spots' X-ray emission is adequately modeled by the SSC process with an equipartition field, while others require an additional component or a lower than equipartition field strength. Suggested explanations have involved (1) a lower magnetic field strength in the X-ray-bright hot spots, which both increases IC emission and increases the loss lifetime of X-ray synchrotron-emitting electrons (Brunetti et al. 2001); or (2) the effects of differential relativistic beaming, due to decelerating bulk motions in the hot spots, on the synchrotron and IC spectra, in particular the fact that fast-moving parts of the flow see the slow-moving downstream flow Doppler boosted (Georganopoulos \& Kazanas 2003), which helps to account for the fact that many of the early detections of X-ray-bright hot spots involved broad-line radio galaxies or quasars (H02), which in unified models should lie relatively close to the line of sight. The small number of published detections of X-ray hot spots has made it difficult to arrive at a definitive answer. 
TABLE 1

3C FR II Sources ObServed with Chandra

\begin{tabular}{|c|c|c|c|c|c|c|c|c|c|}
\hline Source & $z$ & $\begin{array}{l}S_{178} \\
(\mathrm{Jy})\end{array}$ & $\alpha_{r}$ & Galactic $N_{\mathrm{H}}\left(\times 10^{20} \mathrm{~cm}^{-2}\right)$ & $\begin{array}{l}S_{\text {core, }, 5} \\
(\mathrm{mJy})\end{array}$ & Type & Chandra ObsID & $\begin{array}{l}\text { Observing Time } \\
\text { (s) }\end{array}$ & Date Observed \\
\hline 3C $6.1 \ldots \ldots \ldots \ldots$ & 0.8404 & 14.93 & 0.68 & 17.49 & 4.4 & $\mathrm{~N}$ & 3009 & 36492 & 2002 Oct 15 \\
\hline 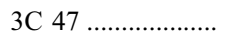 & 0.425 & 28.78 & 0.98 & 5.34 & 73.6 & Q & 2129 & 44527 & 2001 Jan 16 \\
\hline 3C $109 \ldots \ldots \ldots \ldots . . . . .$. & 0.3056 & 23.54 & 0.85 & 14.13 & 263 & B & 4005 & 45713 & 2003 Mar 23 \\
\hline 3C $123 \ldots \ldots \ldots \ldots \ldots . . .$. & 0.2177 & 206.01 & 0.70 & 43 & 100 & $\mathrm{E}$ & 829 & 38465 & 2000 Mar 21 \\
\hline 3C $184 \ldots \ldots \ldots \ldots \ldots . . . .$. & 0.994 & 14.39 & 0.86 & 3.46 & $<0.2$ & $\mathrm{~N}$ & 3226 & 18886 & 2002 Sep 22 \\
\hline 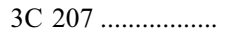 & 0.684 & 14.82 & 0.90 & 5.40 & 510 & Q & 2130 & 37544 & 2000 Nov 04 \\
\hline 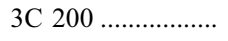 & 0.458 & 12.32 & 0.84 & 3.69 & 35.1 & $\mathrm{~N}$ & 838 & 14660 & 2000 Oct 06 \\
\hline 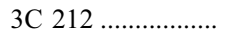 & 1.049 & 16.46 & 0.92 & 4.09 & 150 & Q & 434 & 18054 & 2000 Oct 26 \\
\hline 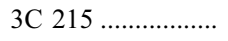 & 0.411 & 12.43 & 1.06 & 3.75 & 16.4 & Q & 3054 & 33803 & 2003 Jan 02 \\
\hline 3C 219 & 0.1744 & 44.91 & 0.81 & 1.48 & 51 & B & 827 & 17586 & 2000 Oct 11 \\
\hline 3C $220.1 \ldots \ldots \ldots \ldots$ & 0.61 & 17.22 & 0.93 & 1.93 & 25 & $\mathrm{~N}$ & 839 & 18922 & 1999 Dec 29 \\
\hline 3C $275.1 \ldots \ldots \ldots \ldots$ & 0.557 & 19.95 & 0.96 & 1.89 & 130 & Q & 2096 & 24757 & 2001 Jun 02 \\
\hline 3C $280 \ldots \ldots \ldots$ & 0.996 & 25.83 & 0.81 & 1.25 & 1.0 & $\mathrm{~N}$ & 2210 & 63528 & 2001 Aug 27 \\
\hline 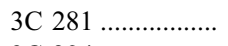 & 0.602 & 6.00 & 0.71 & 2.2 & 19.5 & Q & 1593 & 15851 & 2001 May 30 \\
\hline 3C 294 & 1.78 & 11.23 & 1.07 & 1.20 & 0.53 & $\mathrm{~N}$ & 3207 & 122020 & 2002 Feb 27 \\
\hline 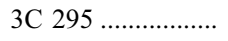 & 0.4614 & 91.02 & 0.63 & 1.38 & 3 & $\mathrm{~N}$ & 2254 & 90936 & 2001 May 18 \\
\hline 3C $303 \ldots \ldots \ldots \ldots$ & 0.141 & 12.21 & 0.76 & 1.60 & 150 & B & 1623 & 14951 & 2001 Mar 23 \\
\hline 3C $321 \ldots \ldots \ldots$ & 0.096 & 14.72 & 0.60 & 4.10 & 30 & $\mathrm{~N}$ & 3138 & 47130 & 2002 Apr 30 \\
\hline 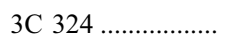 & 1.2063 & 17.22 & 0.90 & 4.47 & $<0.14$ & $\mathrm{~N}$ & 326 & 42147 & 2000 Jun 25 \\
\hline 3C $330 \ldots \ldots \ldots \ldots \ldots . . .$. & 0.5490 & 30.30 & 0.71 & 2.94 & 0.74 & $\mathrm{~N}$ & 2127 & 44083 & 2001 Oct 16 \\
\hline 3C 334 & 0.555 & 11.88 & 0.86 & 4.14 & 111 & Q & 2097 & 32468 & 2001 Aug 22 \\
\hline 3C $351 \ldots \ldots \ldots$ & 0.371 & 14.93 & 0.73 & 2.03 & 6.5 & Q & 2128 & 45701 & 2001 Aug 24 \\
\hline 3C $390.3 \ldots \ldots \ldots \ldots$ & 0.0569 & 51.78 & 0.75 & 3.74 & 330 & B & 830 & 33974 & 2000 Apr 17 \\
\hline 3C $401 \ldots$ & 0.201 & 22.78 & 0.71 & 7.42 & 32 & $\mathrm{E}$ & 3083 & 22666 & 2002 Sep 20 \\
\hline
\end{tabular}

Notes. $-S_{178}$ is the $178 \mathrm{MHz}$ flux density, on the scale of Baars et al. (1977), mostly taken from Laing et al. (1983) or Spinrad et al. (1985); $\alpha_{r}$ is the lowfrequency spectral index, typically between 178 and $750 \mathrm{MHz}$. Types are based on optical and emission-line characteristics and are as follows: E-low-excitation radio galaxy; N-narrow-line radio galaxy; B-broad-line radio galaxy; Q - quasar. Values of $S_{\text {core } 5}$, the $5 \mathrm{GHz}$ core flux density, are mostly taken from the compilation on the 3CRR Web site (http://www.3crr.dyndns.org) or from the radio maps referred to in this paper. All Chandra data sets are from the ACIS-S except 3C 295, where the ACIS-I was used. No grating data met our selection criteria. Live times are the filtered times if filtering was carried out and the uncorrected live time otherwise.

Since the appearance of earlier work aimed explicitly at detecting hot spots (Harris et al. 2000; Hardcastle et al. 2001a; H02; Brunetti et al. 2002), a number of hot spots have been detected in Chandra observations of FR II radio galaxies and quasars made for other purposes (e.g., Donahue et al. 2003; Crawford \& Fabian 2003). This has motivated us to collate all existing data on the hot spots of $3 \mathrm{C}$ radio sources from the Chandra archive and analyze them in a systematic way, with the aim of determining trends and testing models. In this paper we report our results.

Throughout the paper we use a cosmology in which $H_{0}=$ $70 \mathrm{~km} \mathrm{~s}^{-1} \mathrm{Mpc}^{-1}, \Omega_{\mathrm{m}}=0.3$, and $\Omega_{\Lambda}=0.7$. Spectral indices $\alpha$ are the energy indices and are defined in the sense $S_{\nu} \propto \nu^{-\alpha}$.

\section{DATA AND ANALYSIS}

We searched the public Chandra archives for all observations of FR II radio sources in the $3 \mathrm{C}$ catalog made with the CCD Imaging Spectrometer (ACIS), supplementing them with a few observations that we have access to and are not yet public. To make our sample as large as possible, we included any 3C FR II source listed in the archives; this means that we made use of several sources that are not in the better-defined 3CR (Spinrad et al. 1985) or 3CRR (Laing et al. 1983) samples. We restricted ourselves to the $3 \mathrm{C}$ sources simply because they almost all have good radio observations available in the public NRAO Very Large Array (VLA) archives. We excluded compact steep-spectrum sources whose hot spots would not be resolved from the active galactic nucleus (AGN) or lobe emission with Chandra. These selection criteria gave us a sample of 36 sources (Table 1). We next obtained electronic radio maps for all the sources in our sample. Where we did not already have access to a good, high-resolution radio map or to appropriate published radio flux densities, we retrieved the best available data from the VLA archive. In selecting the observations, we preferred data at 5 and $8 \mathrm{GHz}$ in the A configuration (the largest configuration of the VLA), 
TABLE 2

Vla Radio Observations Used in This Paper

\begin{tabular}{|c|c|c|c|c|c|}
\hline Source & Proposal ID & $\begin{array}{l}\text { Frequency } \\
(\mathrm{GHz})\end{array}$ & $\begin{array}{c}\text { Time on Source } \\
(\mathrm{hr})\end{array}$ & Date & $\begin{array}{l}\text { Reference } \\
\text { (if published) }\end{array}$ \\
\hline \multirow[t]{2}{*}{ 3C $6.1 \ldots \ldots \ldots \ldots \ldots \ldots \ldots$} & AP380 & 8.5 & 1 & 1999 Aug 02 & \\
\hline & & 8.5 & $1^{\mathrm{a}}$ & 2000 Jan 18 & \\
\hline 3C 9 & AB369 & 4.9 & 4.5 & 1986 May 04 & 1 \\
\hline 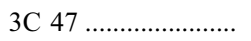 & AB796 & 4.8 & 7 & 1996 Nov 07 & \\
\hline 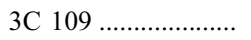 & & 8.4 & & & 2 \\
\hline 3C 123 .................... & & 8.4 & & & 3 \\
\hline 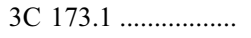 & & 8.4 & & & 3 \\
\hline 3С $179 \ldots \ldots \ldots \ldots \ldots \ldots$ & AC150 & 4.9 & 0.5 & 1986 Mar 21 & \\
\hline 3C 184 ........................ & & 4.9 & & & 4 \\
\hline 3C $200 \ldots \ldots \ldots \ldots \ldots \ldots$ & & 8.4 & & & 2 \\
\hline 3C 207 ......................... & AB796 & 8.5 & 3 & 1996 Nov 08 & \\
\hline 3C 212 & AB796 & 8.5 & 4 & 1996 Nov 07 & \\
\hline 3C $215 \ldots \ldots \ldots \ldots \ldots \ldots$ & & 4.8 & & & 1,5 \\
\hline 3C 219 & & 1.5 & & & 5 \\
\hline 3C $220.1 \ldots \ldots \ldots \ldots \ldots . .$. & & 8.4 & & & 6 \\
\hline 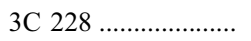 & & 8.4 & & & 2 \\
\hline 3C $254 \ldots \ldots \ldots \ldots \ldots$ & AB522 & 4.9 & 0.5 & 1989 Feb 01 & \\
\hline 3C 263 & & 4.9 & & & 1 \\
\hline 3C 265 & AF186 & 4.8 & 3 & 1990 Apr 22 & 7 \\
\hline 3C $275.1 \ldots \ldots \ldots \ldots \ldots$ & & 8.4 & & & 2 \\
\hline 3C $280 \ldots \ldots \ldots \ldots \ldots \ldots$ & AV157 & 8.4 & 0.7 & 1988 Dec 22 & \\
\hline 3C $281 \ldots \ldots \ldots \ldots \ldots$ & AB631 & 1.4 & 0.3 & 1992 Nov 18 & \\
\hline 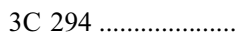 & AM224 & 4.7 & 3.5 & 1987 Oct 11 & \\
\hline 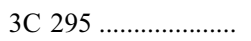 & & 8.4 & & & 2 \\
\hline 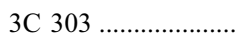 & KRON & 4.9 & $0.3^{\mathrm{b}}$ & 1981 Apr 20 & 8 \\
\hline \multirow[t]{2}{*}{ 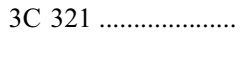 } & AV127 & 4.8 & 3.7 & 1986 Apr 10 & \\
\hline & & $1.5,4.8$ & $2.9,3.7^{\mathrm{a}}$ & 1986 Aug 29 & \\
\hline 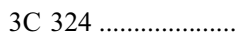 & AF186 & 4.9 & 3 & 1990 Apr 22 & 7 \\
\hline 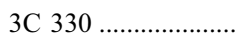 & & 8.4 & & & 2 \\
\hline 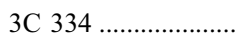 & & 4.9 & & & 1 \\
\hline 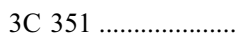 & & 8.4 & & & 2 \\
\hline 3C $390.3 \ldots \ldots \ldots \ldots \ldots \ldots$ & & 1.5 & & & 5 \\
\hline 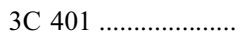 & & 8.4 & & & 3 \\
\hline 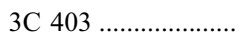 & & 8.4 & & & 9 \\
\hline 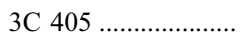 & & 4.5 & & & 10 \\
\hline 3C $427.1 \ldots \ldots \ldots \ldots \ldots . .$. & & 8.4 & & & 2 \\
\hline 3C 438 ......................... & & 8.4 & & & 3 \\
\hline 3C $452 \ldots \ldots \ldots \ldots \ldots . .$. & & 8.4 & & & 9 \\
\hline 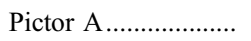 & & 4.9 & & & 11 \\
\hline
\end{tabular}

Notes.-We list VLA observational details only for observations that we have retrieved from the archive and reduced ourselves in the course of this project; for other observations we were able to obtain electronic maps from others (or already had them ourselves), and the reader is referred to the references given below for the observational information. All data retrieved from the archive were taken with the VLA in its A configuration, except where otherwise noted.

${ }^{\mathrm{a}} \mathrm{B}$ configuration data.

${ }^{\mathrm{b}}$ Only one observing frequency of $12.5 \mathrm{MHz}$ bandwidth was used.

REFERENCES.-(1) Bridle et al. 1994; (2) Gilbert et al. 2004; (3) Hardcastle et al. 1997; (4) Belsole et al. 2004; (5) Leahy et al. 1998 (the 3CRR Atlas); (6) Worrall et al. 2001; (7) Fernini et al. 1993; (8) Kronberg 1986; (9) Black et al. 1992; (10) Perley et al. 1984; (11) Perley et al. 1997.

which gives good sensitivity, good separation of lobes and hot spots due to their spectral index differences, and an angular resolution comparable to that of Chandra. Properties of the radio data used and maps made are given in Table 2.

We then used the radio maps as a guide to search for emission from hot spots. We defined a hot spot less strictly than some other workers (e.g., Bridle et al. 1994) both in order to make the comparison between radio and X-ray simpler and because our aim is to include the structure apparently associated with the jet termination whenever possible. In practice, we considered any relatively compact, isolated radio feature that was significantly brighter than its surroundings to be a hot spot (cf. Leahy et al. 1997). However, we excluded any emission that we considered to be associated with a jet; any compact X-ray feature positionally coincident with, or closer to the nucleus than, a linear radio feature that met the definition of Bridle \& Perley (1984) was considered to be a "jet knot" rather than a hot spot. By doing this, we hoped to select only features associated with the termination of the jet and to avoid effects thought to be due to highly relativistic bulk motions, as seen in the X-ray jets of some quasars (e.g., Tavecchio et al. 2000). Later in the paper ( $\$ 5)$ we return to the question of whether hot spots tell us anything about the emission from jet knots or jets in general.

Using the radio data as a guide, we were able to identify a number of previously unreported X-ray counterparts to hot 
spots, in the 10 FR II sources 3C 6.1, 3C 47, 3C 109, 3C 173.1, 3C 228, 3C 321, 3C 324, 3C 334, 3C 403, and 3C 452. The results on 3C 403, 3C 228, and 3C 334 will be reported in more detail elsewhere (3C 403, R. Kraft et al. 2004, in preparation; 3C 228, 3C 334, D. M. Worrall et al. 2004, in preparation); images of the newly detected X-ray hot spots for the other sources are presented in Appendix A. In almost all cases there were a few tens of total counts in the hot spots in the $0.5-$ $5 \mathrm{keV}$ range. Since this is too few to fit spectra, we used the standard Chandra analysis software CIAO to generate redistribution matrix (RMF) and ancillary response (ARF) files appropriate for the hot spots, using the PSEXTRACT tool, corrected for the time-dependent excess ACIS absorption using APPLY_ACISABS, and then used the model-fitting software XSPEC to determine the normalization of a power law, with $\alpha=0.5$ and Galactic absorption, that reproduced the observed net count rate. We chose this power-law index because our aim was to test the validity of the SSC model, which "predicts" $\alpha=0.5$, on the basis of the assumption that the low-energy electron energy index has the value 2.0 associated with particle acceleration at a nonrelativistic strong shock (as appears to be the case in some, although not all, well-studied hot spots; Meisenheimer et al. 1997). However, the choice of $\alpha$ makes relatively little difference to the normalization of the power law and thus to the inferred $1 \mathrm{keV}$ flux density in the observer's frame; using $\alpha=1$ would increase the inferred flux density by between $10 \%$ and $20 \%$. The $1 \mathrm{keV}$ flux densities assuming $\alpha=0.5$ for each source are given in Table 3 .

For the 10 sources 3C 9, 3C 184, 3C 200, 3C 212, 3C 215, 3C 219, 3C 220.1, 3C 401, 3C 427.1, and 3C 438 we found no $\mathrm{X}$-ray emission associated with the hot spots, as previously reported in some cases. Where there was a compact (arcsecond or subarcsecond) radio hot spot present, we determined $3 \sigma$ upper limits on the corresponding X-ray flux density in a 10 pixel detection cell based on Poisson statistics and the local background count rate. Some of these sources (e.g., 3C 401 and 3C 438; Hardcastle et al. 1997) exhibit no compact hot spots in the radio, and we elected not to determine an upper limit on their emission, as the selection of an appropriate X-ray region is difficult. For the same reason, we did not determine an upper limit for the barely resolved source 3C 184, which shows X-ray emission coincident with the radio lobe but not particularly the hot spot (Belsole et al. 2004).

We reexamined previously reported hot spot detections in the 16 sources 3C 123 (Hardcastle et al. 2001a), 3C 179 (Sambruna et al. 2002), 3C 207 (Brunetti et al. 2002), 3C 254 (Donahue et al. 2003), 3C 263 (H02), 3C 265 (Bondi et al. 2003), 3C 275.1 (Crawford \& Fabian 2003), 3C 280 (Donahue et al. 2003), 3C 281 (Crawford \& Fabian 2003), 3C 294 (Fabian et al. 2003), 3C 295 (Harris et al. 2000), 3C 303 (Kataoka et al. 2003), 3C 330 (H02), 3C 351 (Brunetti et al. 2001; H02), 3C 390.3 (Harris et al. 1998), and Cygnus A (3C 405; Harris et al. 1994; Wilson et al. 2000). In all but one case we confirmed the existence of one or more compact X-ray features associated with the radio hot spots (the exception is 3C 281, where the previously reported X-ray emission appears to be diffuse and associated with the lobe and is most likely due to IC scattering of cosmic microwave background [CMB] photons by the lobe rather than SSC from the hot spots). Where a $1 \mathrm{keV}$ flux density had been previously determined from spectral fitting, we make use of that in Table 3. Otherwise, we adopted the same procedure as for the newly detected sources described above. Finally, for all the newly detected and known sources, we determined upper limits, again as described above, for any compact hot spots that were not detected (e.g., in the lobe on the opposite side of the nucleus to the known hot spot). In the process of doing this we found one additional hot spot, $3 \mathrm{C} 123 \mathrm{~W}$, that was formally significantly detected; although the situation in this source is confused by the presence of strong, unrelaxed cluster emission (Hardcastle et al. 2001a), we added it to the sample as a detection, for consistency with the other sources. Because of its intrinsic interest and the extreme nature of its X-ray hot spot, we added Pictor A (Wilson et al. 2001) to the sample (it is not in $3 \mathrm{C}$ because of its low declination but meets the other selection criteria). The overall final sample thus contains 37 sources (Table 1) with 65 X-ray hot spot flux densities or upper limits. All the fluxes and upper limits are tabulated in Table 3.

Finally, for the sources with detected X-ray hot spots, we used the Hubble Space Telescope (HST) archive to search for optical counterparts. Optical emission is important because it constrains the spectrum between radio and X-ray; early work on SSC hot spots was supported by the observation that a onezone synchrotron model could not be fitted through the radio and X-ray data points because of the optical constraints (e.g., Harris et al. 1994). We identified two new candidate optical hot spot counterparts (in 3C 228 and 3C 275.1) and measured flux densities or upper limits for a number of other sources, using the IRAF package SYNPHOT to calculate the conversion factor between observed counts and flux density. Optical flux densities and frequencies are tabulated in Table 4. Sources where there were no archival HST observations, where the hot spot did not lie on the WFPC2 CCDs, or where observational constraints such as a nearby bright star or cosmic-ray contamination prevented us from obtaining a flux density are not tabulated. We also tabulate a number of flux densities, largely based on ground-based observations, taken from other papers, either in the literature or in preparation.

\section{MODELING AND RESULTS}

The large number of detected X-ray hot spots is interesting in itself, given that calculations based on SSC emission at equipartition suggested that only the few brightest hot spots would be detected with Chandra (e.g., Hardcastle 2001). In order to assess quantitatively the extent to which the new detections conflict with an SSC model, we decided to fit a simple SSC model to all the hot spots and determine the ratio between the observed and predicted flux densities. We carried out this calculation using the code of Hardcastle et al. (1998); a brief sketch of the operation of this code is given in Appendix B. The code assumes a spherically symmetric, homogenous hot spot with an electron energy spectrum that can be described as a power law or broken power law. To determine the radius of the hot spot, we therefore fitted models consisting of a homogeneous sphere convolved with the restoring beam to the highest resolution radio data available, in the manner described by $\mathrm{H} 02$. Where multifrequency radio data were available for the hot spot, which was only true in the best-studied cases, we used them to fit a two-component power-law model with an energy spectral break corresponding to $\Delta \alpha=0.5$ (Heavens \& Meisenheimer 1987); otherwise, we assumed a single power law with $\alpha=0.5$ extending from the radio into the millimeterwave regime. Unless good low-frequency radio constraints were available, we assumed that the minimum Lorentz factor of the electrons, $\gamma_{\min }$, was $1000 ; \gamma_{\max }$ was chosen to ensure that there was no spectral cutoff before the millimeter-wave region. These choices reflect what has been found in the best-studied hot spots but are clearly no substitute for good, multifrequency 
TABLE 3

Radio and X-Ray Flux Densities and Predicted Inverse Compton Flux Densities

\begin{tabular}{|c|c|c|c|c|c|c|}
\hline Source & Hot Spot & $\begin{array}{l}\text { Angular Size } \\
\quad(\operatorname{arcsec})\end{array}$ & $\begin{array}{c}5 \text { GHz Radio Flux } \\
\text { (Jy) }\end{array}$ & $\begin{array}{c}1 \mathrm{keV} \text { Flux } \\
(\mathrm{nJy})\end{array}$ & $\begin{array}{l}\text { Predicted Flux } \\
\text { (nJy) }\end{array}$ & $\begin{array}{c}\text { Ratio } R \\
\text { (observed/predicted) }\end{array}$ \\
\hline \multirow[t]{2}{*}{ 3C 6.1} & $\mathrm{~N}$ & 0.36 & 0.340 & 0.45 & 0.19 & 2.3 \\
\hline & $\mathrm{S}$ & 0.41 & 0.200 & 0.09 & 0.081 & 1.1 \\
\hline \multirow[t]{2}{*}{ 3C 9} & $\mathrm{~N}$ & 0.38 & 0.038 & $<0.09$ & 0.024 & $<3.6$ \\
\hline & $\mathrm{S}$ & 0.39 & 0.012 & $<0.09$ & 0.0046 & $<20$ \\
\hline \multirow[t]{2}{*}{ 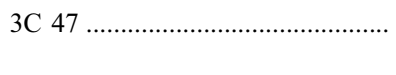 } & $\mathrm{S}$ & 0.434 & 0.181 & 0.54 & 0.040 & 14 \\
\hline & $\mathrm{N}$ & 1.89 & 0.127 & $<0.1$ & 0.015 & $<6.6$ \\
\hline \multirow[t]{2}{*}{ 3C 109} & $\mathrm{~S}$ & 0.377 & 0.181 & 0.15 & 0.033 & 4.6 \\
\hline & $\mathrm{N}$ & 0.274 & 0.007 & $<0.09$ & 0.00053 & $<169$ \\
\hline \multirow[t]{2}{*}{$3 \mathrm{C} 123$} & $\mathrm{E}$ & $1.1 \times 0.54$ & 5.12 & 4.6 & 2.2 & 2.1 \\
\hline & $\mathrm{W}$ & $1.0 \times 0.13$ & 0.341 & 0.18 & 0.059 & 3.1 \\
\hline \multirow[t]{2}{*}{ 3С 173.1} & $\mathrm{~S}$ & 0.83 & 0.033 & 0.2 & 0.0022 & 91 \\
\hline & $\mathrm{N}$ & 0.26 & 0.009 & $<0.12$ & 0.00051 & $<237$ \\
\hline \multirow[t]{2}{*}{ 3C 179} & $\mathrm{~W}$ & 0.145 & 0.063 & 1.54 & 0.026 & 63 \\
\hline & $\mathrm{E}$ & 0.45 & 0.038 & $<0.26$ & 0.0070 & $<37$ \\
\hline 3C 200 & $\mathrm{~N}$ & 0.6 & 0.057 & $<0.1$ & 0.011 & $<9.5$ \\
\hline \multirow{3}{*}{$3 \mathrm{C} 212$} & $\mathrm{E}$ & 0.27 & 0.044 & 0.69 & 0.0095 & 73 \\
\hline & $\mathrm{N}$ & 0.144 & 0.035 & $<0.14$ & 0.014 & $<10$ \\
\hline & $\mathrm{S}$ & 0.25 & 0.110 & $<0.14$ & 0.056 & $<2.5$ \\
\hline 3C 215 & $\mathrm{E}$ & 1.0 & 0.012 & $<0.04$ & 0.0011 & $<36$ \\
\hline \multirow[t]{2}{*}{ 3C $220.1 \ldots \ldots \ldots \ldots \ldots \ldots \ldots \ldots \ldots \ldots \ldots \ldots \ldots$} & $\mathrm{E}$ & 0.27 & 0.021 & $<0.13$ & 0.0025 & $<52$ \\
\hline & $\mathrm{W}$ & 0.27 & 0.023 & $<0.13$ & 0.0030 & $<44$ \\
\hline \multirow[t]{2}{*}{ 3C 228} & $\mathrm{~N}$ & 0.203 & 0.070 & 0.45 & 0.019 & 24 \\
\hline & $\mathrm{S}$ & 0.265 & 0.132 & 1.3 & 0.042 & 31 \\
\hline 3C 254 & $\mathrm{~W}$ & 0.29 & 0.146 & 0.54 & 0.061 & 8.8 \\
\hline \multirow[t]{2}{*}{ 3C 263} & $\mathrm{E}$ & 0.39 & 0.582 & 1.0 & 0.25 & 4.0 \\
\hline & $\mathrm{W}$ & 0.18 & 0.023 & $<0.06$ & 0.0054 & $<11$ \\
\hline \multirow[t]{2}{*}{ 3C 265} & $\mathrm{E}$ & 0.356 & 0.272 & 0.35 & 0.16 & 2.2 \\
\hline & $\mathrm{W}$ & 0.73 & 0.048 & 0.13 & 0.088 & 15 \\
\hline \multirow[t]{2}{*}{ 3C 275.1} & $\mathrm{~N}$ & $1.4 \times 0.2$ & 0.191 & 1.78 & 0.093 & 19 \\
\hline & $\mathrm{S}$ & 0.378 & 0.111 & $<0.12$ & 0.038 & $<3.1$ \\
\hline \multirow[t]{3}{*}{$3 \mathrm{C} 280$} & $\mathrm{E}$ & 0.186 & 0.082 & 0.31 & 0.046 & 6.7 \\
\hline & W & 0.146 & 0.631 & 0.6 & 1.2 & 0.48 \\
\hline & Wc & 0.081 & 0.035 & 0.07 & 0.021 & 3.35 \\
\hline 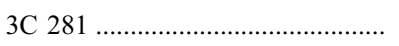 & $\mathrm{N}$ & 1.04 & 0.129 & $<0.16$ & 0.022 & $<7.3$ \\
\hline \multirow[t]{2}{*}{ 3C 294} & $\mathrm{~N}$ & 0.283 & 0.143 & 0.12 & 0.17 & 0.72 \\
\hline & $\mathrm{S}$ & 0.43 & 0.022 & 0.14 & 0.0088 & 16 \\
\hline \multirow{2}{*}{ 3C 295} & $\mathrm{~N}$ & 0.1 & 1.29 & 1.4 & 0.78 & 1.8 \\
\hline & $\mathrm{S}$ & 0.1 & 0.92 & 0.94 & 0.85 & 1.1 \\
\hline 3С 303 & $\mathrm{~W}$ & $1.1 \times 0.28$ & 0.257 & 4.0 & 0.026 & 154 \\
\hline & $\mathrm{E}$ & 0.57 & 0.0025 & $<0.16$ & 0.00039 & $<408$ \\
\hline 3C $321 \ldots \ldots$ & $\mathrm{E}$ & 0.69 & 0.125 & 0.3 & 0.006 & 48 \\
\hline & $\mathrm{W}$ & $2.7 \times 0.45$ & 0.020 & 0.12 & 0.00057 & 210 \\
\hline 3C 324 & $\mathrm{E}$ & 0.365 & 0.277 & 0.20 & 0.21 & 0.93 \\
\hline & W & 0.301 & 0.085 & 0.16 & 0.040 & 4.04 \\
\hline 3C 330 & $\mathrm{~N}$ & 0.45 & 0.625 & 0.35 & 0.42 & 0.81 \\
\hline & $\mathrm{S}$ & 0.20 & 0.102 & 0.068 & 0.028 & 2.4 \\
\hline 3C 334 & $\mathrm{~S}$ & $1.34 \times 0.3$ & 0.018 & 0.54 & 0.0018 & 292 \\
\hline & $\mathrm{N}$ & 0.5 & 0.007 & $<0.4$ & 0.00066 & $<604$ \\
\hline 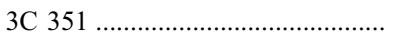 & $\mathrm{J}$ & 0.16 & 0.167 & 4.3 & 0.051 & 85 \\
\hline & $\mathrm{K}$ & 0.8 & 0.406 & 3.4 & 0.087 & 39 \\
\hline & $\mathrm{S}$ & 0.16 & 0.0025 & $<0.05$ & 0.00014 & $<362$ \\
\hline 3C 390.3 & $\mathrm{~N}$ & $1.3 \times 0.5$ & 0.087 & 4.5 & 0.003 & 1380 \\
\hline & $\mathrm{S}$ & 3.7 & 1.30 & 1.3 & 0.07 & 19 \\
\hline 3C 403 & $\mathrm{~F} 1$ & 0.275 & 0.021 & 1.0 & 0.00047 & 2149 \\
\hline & F6 & 0.256 & 0.035 & 1.8 & 0.0013 & 1414 \\
\hline 3C 405 & A & $2.5 \times 1.25$ & 38.0 & 19.4 & 15.5 & 1.3 \\
\hline & $\mathrm{B}$ & 0.44 & 3.04 & 4.5 & 0.71 & 6.3 \\
\hline & $\mathrm{D}$ & 1.09 & 30.3 & 29.2 & 13.6 & 2.1 \\
\hline & $\mathrm{E}$ & $0.45 \times 0.63$ & 1.68 & 1.2 & 0.23 & 5.2 \\
\hline
\end{tabular}


TABLE 3-Continued

\begin{tabular}{|c|c|c|c|c|c|c|}
\hline Source & Hot Spot & $\begin{array}{l}\text { Angular Size } \\
\quad(\operatorname{arcsec})\end{array}$ & $\begin{array}{l}5 \mathrm{GHz} \text { Radio Flux } \\
\text { (Jy) }\end{array}$ & $\begin{array}{c}1 \mathrm{keV} \text { Flux } \\
\text { (nJy) }\end{array}$ & $\begin{array}{l}\text { Predicted Flux } \\
\text { (nJy) }\end{array}$ & $\begin{array}{c}\text { Ratio } R \\
\text { (observed/predicted) }\end{array}$ \\
\hline \multirow{2}{*}{ 3C 427.1} & $\mathrm{~N}$ & 0.165 & 0.019 & $<0.17$ & 0.0030 & $<56$ \\
\hline & $\mathrm{S}$ & 0.14 & 0.025 & $<0.17$ & 0.0046 & $<37$ \\
\hline \multirow[t]{2}{*}{$3 \mathrm{C} 452$} & W & 0.705 & 0.033 & 0.34 & 0.00095 & 356 \\
\hline & $\mathrm{E}$ & 3.0 & 0.067 & $<0.05$ & 0.0039 & $<13$ \\
\hline \multirow[t]{2}{*}{ Pic A } & $\mathrm{W}$ & 0.75 & 1.93 & 89 & 0.20 & 454 \\
\hline & $\mathrm{E}$ & 0.75 & 0.467 & $<0.16$ & 0.044 & $<6.4$ \\
\hline
\end{tabular}

Notes.-The hot spot identifier is usually N, S, E, or W, referring to the obvious or brightest hot spot in the north, south, east, or west lobes. Exceptions are made where a multiple-hot spot source has names for the individual components that are used relatively widely in the literature; this is true of 3C 405 (notation of Hargrave \& Ryle 1974), 3C 351 (notation of Bridle et al. 1994), and 3C 403 (notation of Black et al. 1992). The angular sizes quoted are the radii of homogeneous sphere models fitted to the radio data, as described in the text, except where two numbers are quoted, in which case they are the length and radius of a cylinder and are generally directly measured from high-resolution maps. The measured hot spot radio flux densities have been scaled to a laboratory frame radio frequency of $5 \mathrm{GHz}$ using a spectral index $\alpha=0.5$ for ease of comparison. The $1 \mathrm{keV}$ flux densities are the values inferred from spectral fitting or the observed count rate, as described in the text, and are the unabsorbed fluxes (assuming Galactic absorption).

TABLE 4

Optical Flux Densities Used in This Paper and Associated HST Observational Details

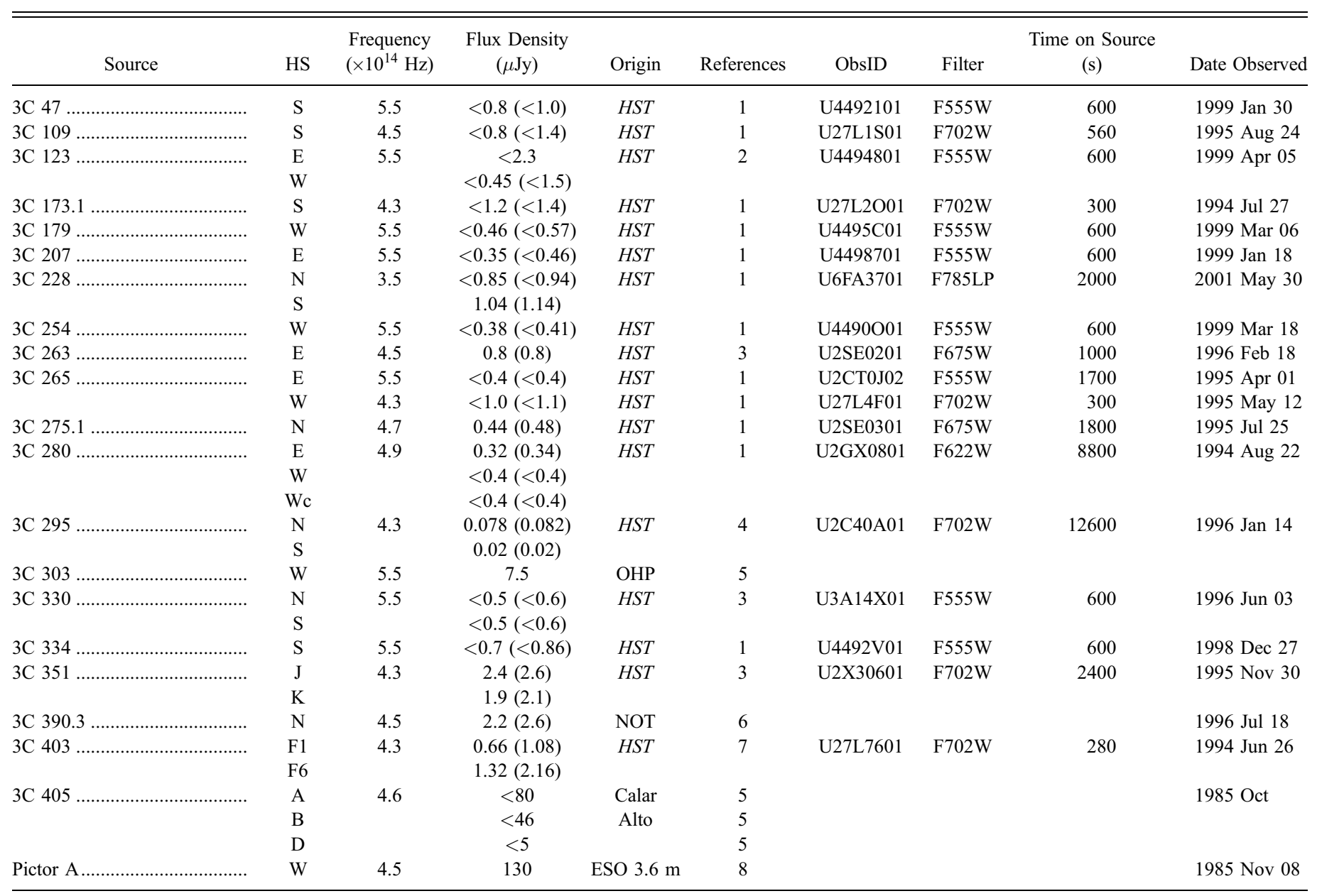

Notes.- Hot spots are identified as in Table 3. Optical flux densities are corrected for Galactic extinction if only one value is given; where two are given the second (in parentheses) is the corrected value. HST observational details are given where HST data were used; other data points are taken from ground-based observations described in the literature. Data points for the same object have the same origin unless different origins are explicitly listed in the table. NOT indicates the Nordic Optical Telescope and OHP the Observatoire de Haute Provence.

References.- (1) This paper; (2) Hardcastle et al. 2001a; (3) H02; (4) Harris et al. 2000; (5) Meisenheimer et al. 1997; (6) Harris et al. 1998; (7) R. Kraft et al. 2004, in preparation; (8) Röser \& Meisenheimer 1987. 

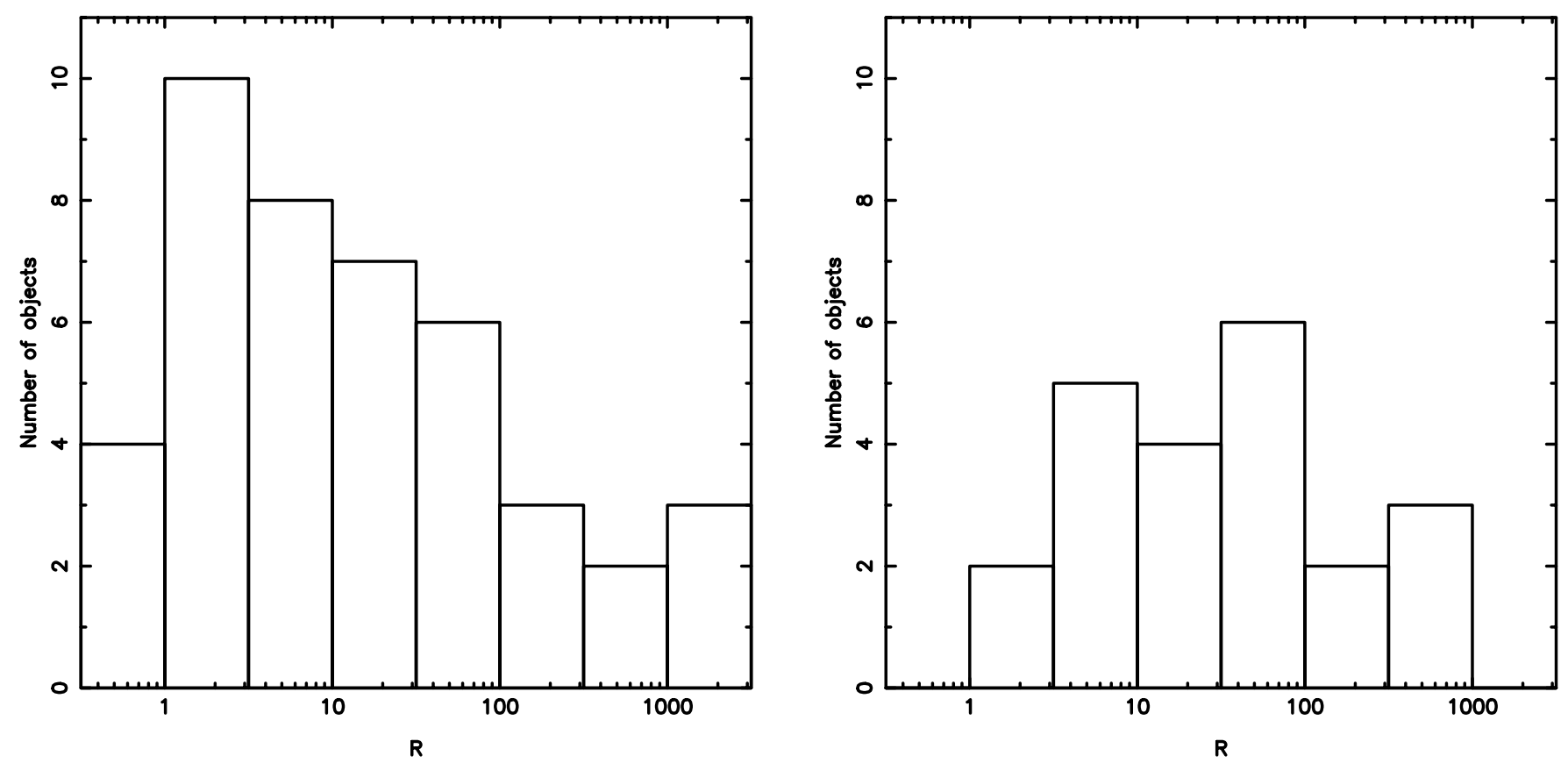

FIG. 1.-Distribution of the ratio $R$ of observed X-ray flux to IC prediction for the Chandra sample. Left: Distribution for X-ray-detected hot spots. Right: Distribution for compact hot spots with no X-ray detection (sources can move to the left).

observations, particularly at high frequencies. However, we estimate that these choices make a difference at the level of at most $\sim 10 \%-20 \%$ (except in the rare cases where a $\sim 10 \mathrm{GHz}$ spectral cutoff is present). The results are particularly insensitive to the choice of $\gamma_{\min }$, since decreasing this has two effects that act in opposite directions: more high-energy photons are scattered by the large additional population of low-energy electrons, but the overall electron energy power-law normalization is reduced to maintain equipartition. Detailed spatial modeling, where high-resolution observations make it possible, also changes the results of SSC calculations at the $10 \%-20 \%$ level (H02), so that overall the calculated value should be a good estimate of the true inverse Compton prediction. The equipartition flux density prediction (taking into account both SSC and IC scattering of CMB photons) and the ratio $R$ between the observed and predicted flux densities are tabulated in Table 3 for each hot spot. Note that in almost all cases the flux due to the SSC process dominates over that due to IC scattering of the CMB (assuming no relativistic beaming) by an order of magnitude or more. For simplicity we often refer to the calculated fluxes as SSC fluxes in what follows.

The tabulated values of $R$ are calculated assuming that there are no protons, so that equipartition is between the radiating electrons and magnetic fields only, and that the filling factor of the hot spots is unity. If we were to include an energetically dominant population of protons in equipartition in our model, it would reduce the predicted inverse Compton emission (since the number of electrons decreases) and so increase the ratio $R$, possibly by a large factor. If the ratio of proton to electron energy densities were in the ratio of their rest masses, $R$-values would increase by about a factor of 30-70. Even if the proton-to-electron energy densities were of the order of their number ratios as observed in cosmic rays at Earth (an energy-dependent factor of 50-100; Longair 1992), we would expect $R$ to increase by a factor of $\sim 5-10$. A population of protons with the same total energy as the electrons has a less dramatic effect, increasing $R$ by only about $40 \%$. In any case, it is clear that introducing protons cannot solve the problem of high- $R$ hot spots. The predicted SSC inverse Compton emission can be increased, and $R$ can be decreased, if we have overestimated the volume or the filling factor, although (depending on the space-filling fluid) the actual results of a low filling factor can be very geometry dependent: if the electrons are confined to thin sheets, so that the probability of scattering is comparatively low, then the effects of low filling factor can be less than expected. Roughly (see Appendix B), to reduce $R$ by a factor of 1000 , and so to make the most extreme observed hot spots consistent with being inverse Compton emission at equipartition, we would need to reduce the volume or the filling factor by a factor of $\sim 10^{12}$, and this neglects geometrical effects. Such low filling factors are clearly implausible.

Figure 1 shows the distribution of the ratio $R$ and upper limits on $R$ for the sample. Two points are immediately obvious: first, the detected X-ray flux density lies significantly above the IC prediction in most sources; second, there are few detected sources with $R<1$, and there is a clear change in the distribution of sources at around $R=1$. If the upper limits on $\mathrm{X}$-ray flux for the nondetected sources all lie a long way above the true values, then this could change, but if it does not, the special status of $R=1$ implies that few sources have less X-ray emission than would be expected on the equipartition $\mathrm{SSC} / \mathrm{IC}$ model. This could suggest either that all sources have SSC/IC X-ray emission at a level consistent with the equipartition prediction, together with some additional source of X-ray emission, or that there are departures from equipartition (some quite large) but that these are always in the sense that $B<B_{\text {eq }}$. The fact that we do not see many sources with $R \ll 1$ suggests that there are few or no hot spots with $B \gg B_{\text {eq }}$, although without detections of all the hot spots we cannot be more definite.

If the model we have used to predict the level of inverse Compton emission is incorrect, then the special status of $R=1$ would have to be a coincidence. This, as we have argued before 


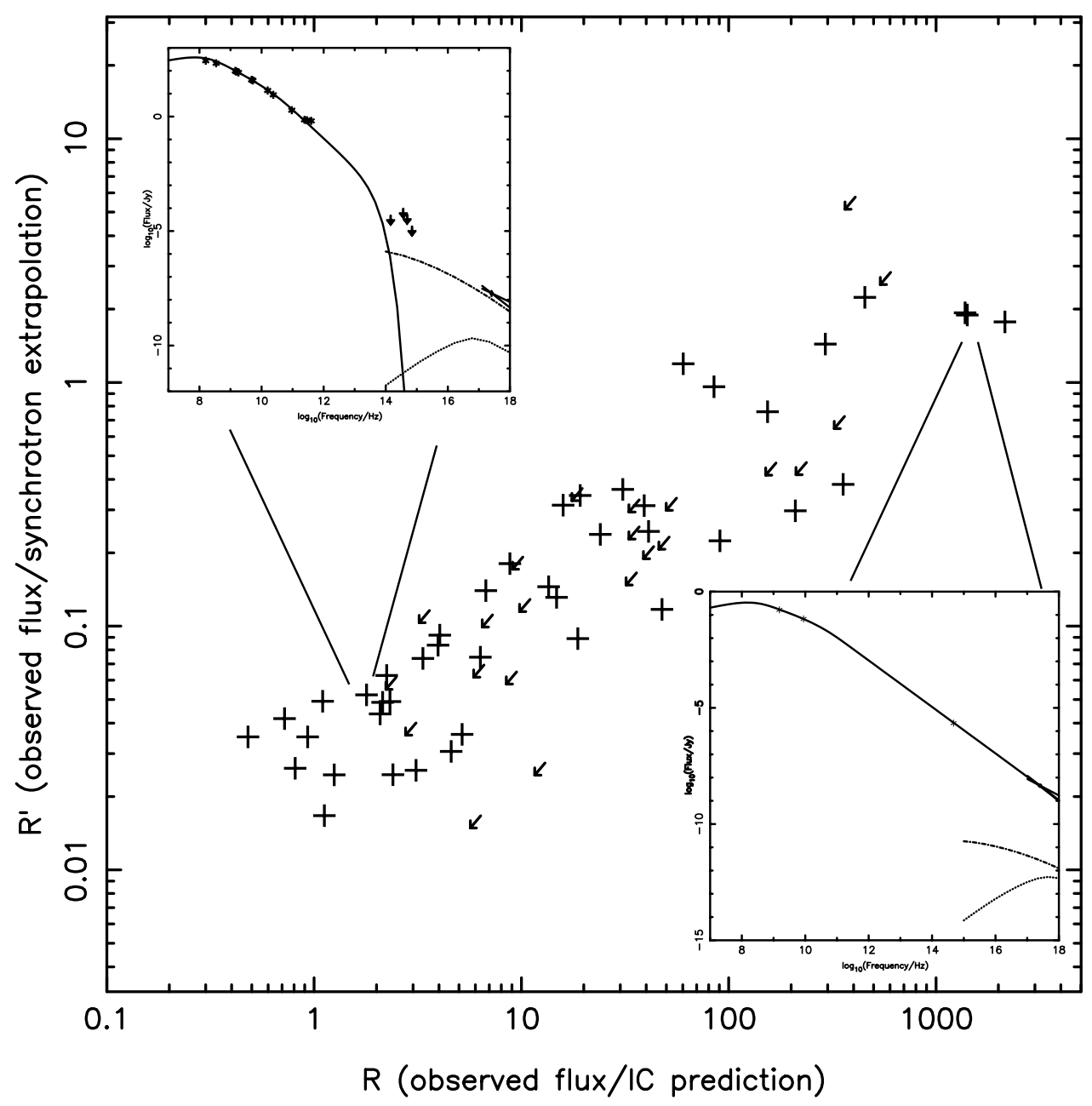

FIG. 2. $-R^{\prime}$ is plotted against $R$, where $R$ is the ratio between the observed X-ray flux density and the prediction of an inverse Compton model at equipartition, and $R^{\prime}$ is defined as the ratio of the observed flux to the extrapolation of the radio flux density assuming $\alpha=1.0$, i.e., to the amount of X-rays that could (conservatively) have been produced by synchrotron emission with a straight spectrum. Diagonal arrows show upper limits from nondetected X-ray sources. Insets show the broadband SEDs of two extreme sources on the plot: Cygnus A hot spot A (top left) and 3C 390.3 N hot spot (bottom right). The data points are from the literature or from maps available to us, the solid line represents the best-fitting synchrotron model, and the dot-dashed and dotted lines represent the equipartition SSC and CMB inverse Compton models, respectively.

(e.g., H02), gives us a reason to disfavor models with an energetically dominant proton population, or with consistently very low filling factor. Thus, for example, if the proton-toelectron energy density ratio were $\sim 100$, we would expect a source in equipartition, and emitting in X-rays only via the SSC process, to have $R \approx 0.1-0.2$ given our model (since we would be overpredicting the inverse Compton emission); we would not see a special status for $R=1$ unless other parameters (such as filling factor) conspired systematically to increase the value of $R$, which is inherently improbable. We emphasize that, given the small numbers and the width of the distribution of $R$-values, this does not rule out moderate filling factors or a proton population within a factor of $\sim 10$ of the energy density in the other components.

What emission mechanisms are possible for the detected hot spots? We began by calculating another parameter, $R^{\prime}$, the ratio between the observed X-ray flux density and the flux predicted from a simple power-law extrapolation (with $\alpha_{\mathrm{rx}}=1.0$ ) from the radio data. Hot spots with both $R \gg 1$ and $R^{\prime} \gg 1$ would represent a problem for both synchrotron and inverse Compton models. However, we find that $R^{\prime}$ is almost always less than 1, so there are no sources whose X-ray flux is impossible to explain with a synchrotron model in this sense.
The plot of $R^{\prime}$ against $R$ (Fig. 2) shows that even the most extreme X-ray hot spots, in terms of $R$, can readily be accounted for with a synchrotron model. There is a smooth distribution in parameter space, with no obvious bimodality, between sources with $R^{\prime}=1, R \gg 1$ (where a synchrotron model is natural) and $R=1, R^{\prime} \ll 1$ (where an inverse Compton model has tended to be adopted in earlier work). Insets in Figure 2 show the very different SEDs of sources at the extreme ends of the distribution and also illustrate the importance of optical constraints in determining the X-ray emission mechanism. To investigate this further, we used the available optical data or upper limits for X-ray-detected hot spots to constrain their spectral shape. We calculated the quantities $\alpha_{\text {ro }}$ and $\alpha_{\text {ox }}$, the two-point radio-to-optical and optical-to-X-ray spectral indices, for all the X-ray-detected hot spots with optical flux densities or upper limits. A hot spot in which the optical-to-X-ray spectrum is flatter than the radio-to-optical spectrum $\left(\alpha_{\mathrm{ox}}<\alpha_{\mathrm{ro}}\right)$ cannot be described by a simple one-zone synchrotron model in which the spectrum steepens with increasing frequency. The difference between the two spectral indices is plotted in Figure 3 as a function of $R$. It can be seen that the more extreme hot spots (large $R$-values) all have $\alpha_{\text {ox }}-\alpha_{\text {ro }}>0$ and so are consistent with a 


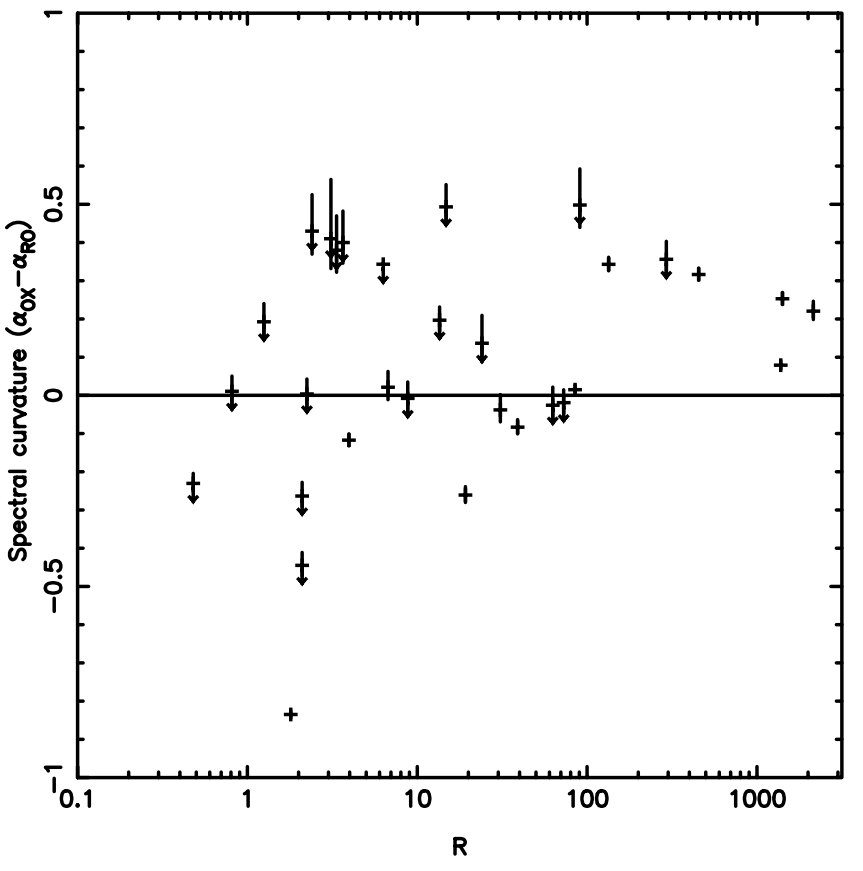

FIG. 3.- Spectral index difference (curvature indicator) against $R$ for sources with optical hot spots or upper limits on optical flux. The error bars show the statistical errors on X-ray flux density only, as these are the dominant errors.

synchrotron model. A nonsynchrotron model is required by the optical data only for a few low- $R$ objects, where inverse Compton emission is the accepted and most plausible X-ray mechanism. The existing data do not rule out a model in which synchrotron X-ray emission is important in a significant number of our target objects, although this is far from conclusive given the large number of optical nondetections.

What determines the value of $R$ for a particular hot spot? We noted that the early detections of SSC emission, such as Cygnus A, 3C 295, and 3C 123, were all in luminous sources, while well-studied problematic sources such as 3C 390.3 and Pictor A are much lower in overall radio luminosity. Accordingly, we looked for a relationship between $R$ and total $178 \mathrm{MHz}$ luminosity from the original $3 \mathrm{C}$ measurements or the revised values of Laing et al. (1983) (correcting by a factor 1.09 so as to bring the flux densities on to the scale of Baars et al. 1977, and using low-frequency spectral indices to correct to the rest frame), obtaining the plot shown in Figure 4. The inverse correlation seen here appears to indicate a role of the source luminosity in determining $R$. The correlation is improved if we plot the luminosity of only the hot spot against $R$ (Fig. 5); here we have used measurements from the radio maps, correcting to a rest-frame frequency of $5 \mathrm{GHz}$ by assuming a radio spectral index of 0.5 . The improvement suggests that the relationship with hot spot luminosity is primary and that the correlation with overall source luminosity arises because of the correlation between hot spot and source luminosity. It is important to realize that these plots are not necessarily an indication of a one-to-one correlation between $R$ and hot spot or total source luminosity. First, Chandra's sensitivity (around $0.1 \mathrm{nJy}$ at $1 \mathrm{keV}$ for the exposure times used in these observations) means that we would not expect to detect the SSC emission from the lowest luminosity hot spots, so that it is observationally impossible to populate the bottom left corner of Figure 5, as shown by the dotted lines illustrating the observational limits. Second, there is a positive

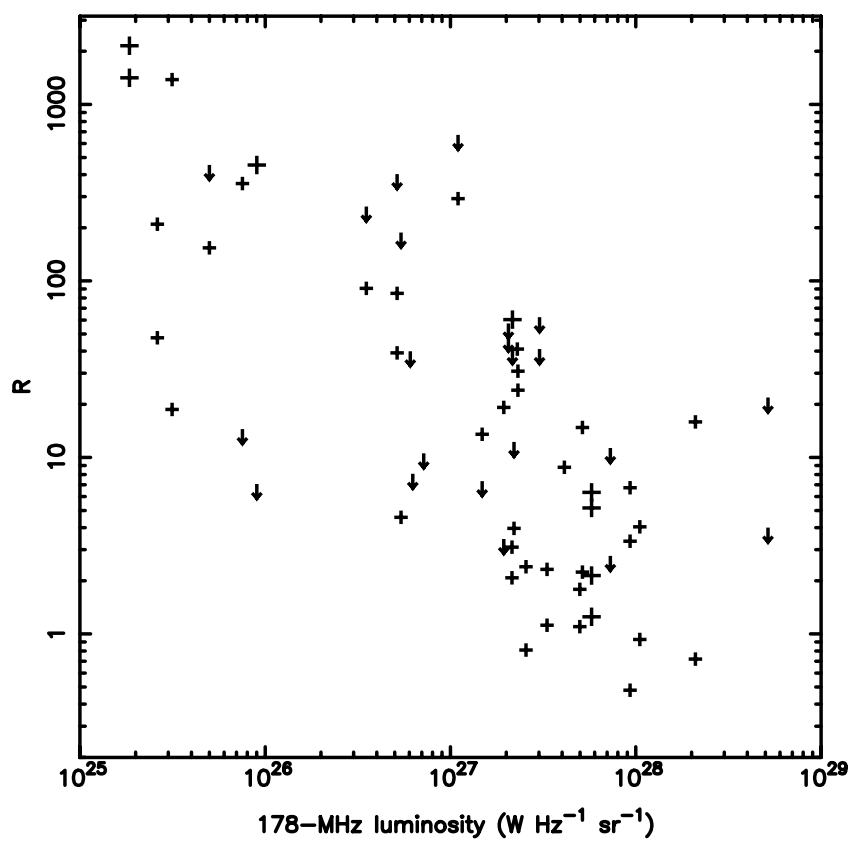

FIG. 4. $-R$ plotted against the total rest-frame $178 \mathrm{MHz}$ luminosity, from the $3 C / 3 C R R$ measurements. Since most sources have more than one hot spot, typically two $R$-values are plotted for a given source luminosity.

correlation between the predicted SSC flux density and the hot spot luminosity, since the sample is flux limited and the SSC luminosity is a nonlinear function of the hot spot luminosity (for a given hot spot size), and this increases the strength of the apparent correlation. However, there is at least one key result from this analysis: there are no hot spots with high luminosity and high $R$; we would certainly have been able to detect such hot spots if they existed. By contrast, lowluminosity hot spots appear to be able to have extremely high

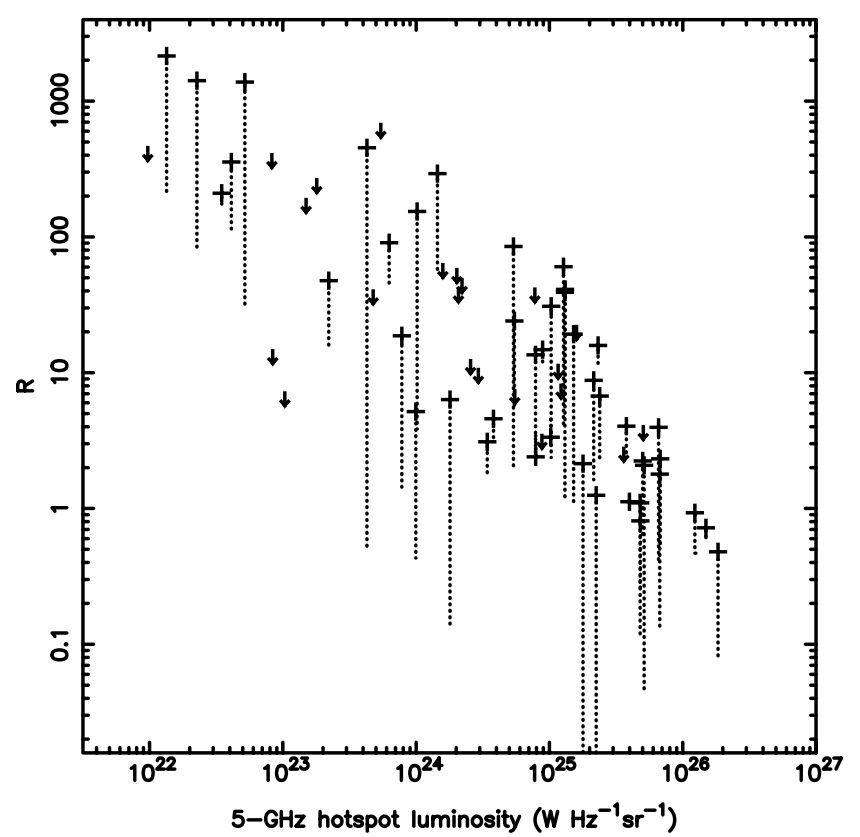

FIG. 5. $-R$ plotted against the rest-frame $5 \mathrm{GHz}$ luminosity of the hot spot. The dotted lines extending down from the data points show the approximate lowest value of $R$ that could have been detected with the data, assuming a nominal Chandra sensitivity of $0.1 \mathrm{nJy}$ at $1 \mathrm{keV}$. 

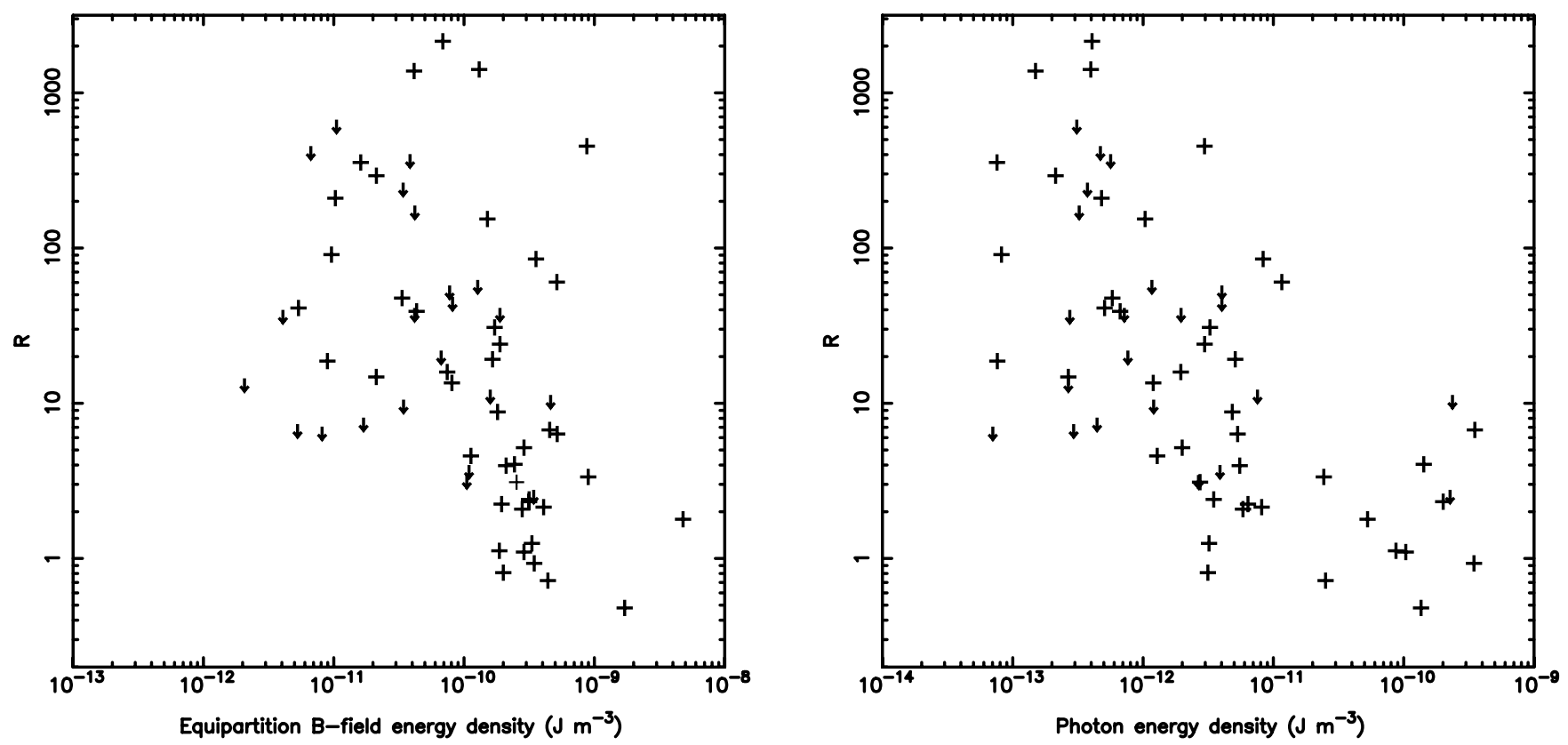

FIG. 6. $-R$ plotted against the magnetic field and photon energy densities in the hot spots. The plotted photon energy density takes into account both synchrotron and CMB photons. Note that the two plots have different scales on the $x$-axis.

$R$-values, although we cannot say definitely that all of them do. We find no other relationships between $R$ and hot spot or source parameters such as hot spot angular or linear size, source size, redshift, or radio spectral index. However, there are relationships between $R$ and other derived quantities such as equipartition magnetic field energy density and photon energy density (Fig. 6); these are not surprising, since all of the quantities are related to radio luminosity. We return to the possible physical significance of these relationships below (§ 4.2).

Finally, we investigated the role of beaming in determining the X-ray brightness of hot spots by plotting the $R$ parameter against the core prominence, defined here as the ratio of $5 \mathrm{GHz}$ core flux density to (rest frame) $178 \mathrm{MHz}$ total source flux density. Core prominence is often used as a proxy of beaming (e.g., Orr \& Browne 1982; Kapahi \& Murphy 1990; Morganti et al. 1997; Hardcastle et al. 1999), relying on the assumption that the intrinsic fraction of the radio source flux emitted by the core is similar in all sources and that the observed variation in core prominence arises from relativistic beaming in the parsecscale jet. Using the core flux densities tabulated in Table 1, we produced the plot shown in Figure 7. This figure certainly shows a trend, in the sense that (as was already clear) many of the X-ray-overbright hot spots are in beamed sources and often on the same side as a known one-sided radio jet. At the same time, there is clearly a good deal of scatter in any correlation (up to 2.5 orders of magnitude separate sources with similar core prominences), and there are sources that do not fit it at all, such as the low core-prominence, narrow-line source 3C $403(R>1000)$. If we plot core prominence against hot spot radio luminosity (Fig. 8), we see that there is a tendency for sources with high core prominences to have low-luminosity hot spots, a trend that can be explained entirely in terms of a bias toward broad-line radio galaxies and quasars at low redshifts in the parent sample, so that it is not clear that the trend seen in Figure 7 is meaningful. Although the quasars and broad-line radio galaxies (in unified models, the sources that should be most strongly affected by beaming) tend to lie at the upper edge of the envelope of $R$-values for a given luminosity range, the dominant effect is the luminosity dependence. A partial Kendall's $\tau$ analysis taking into account the upper limits, performed according to the prescription of Akritas \& Siebert (1996) and using their code, shows that the correlation between $R$ and core prominence is not significant at the $95 \%$ confidence level if the luminosity correlation is taken into account, while the correlation between $R$ and luminosity is significant even given the core prominence relation. Thus, with the current data, we have no significant evidence for a relationship between hot spot $R$-value and beaming, and the

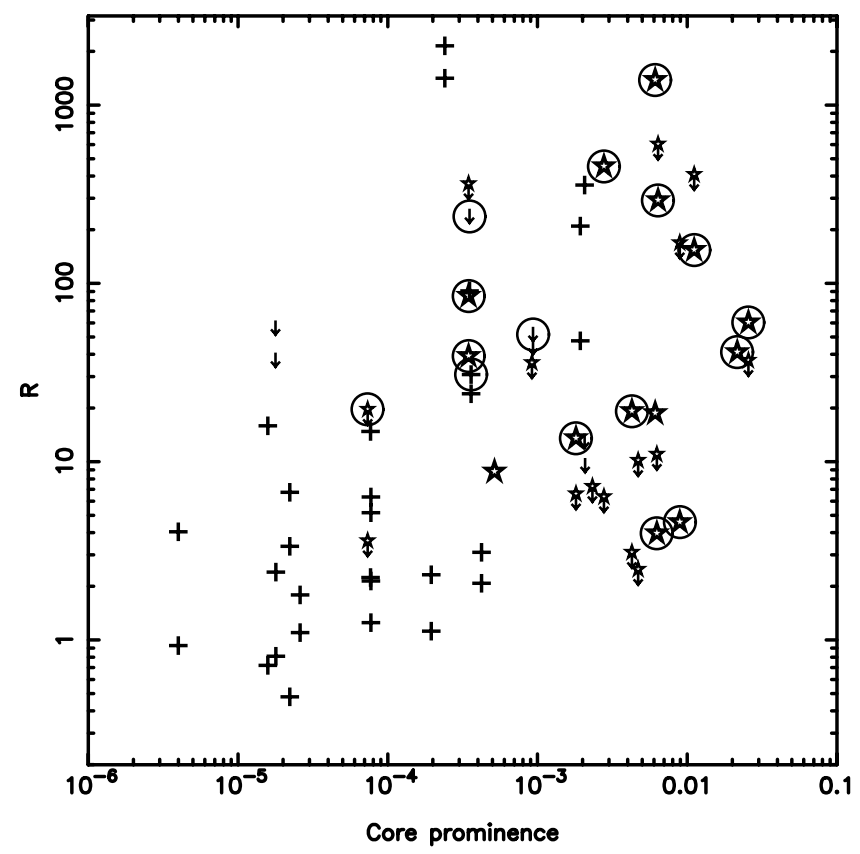

FIG. 7. $-R$ plotted against core prominence. Stars indicate broad-line objects (broad-line radio galaxies and quasars) that are expected to lie at angles $\lesssim 45^{\circ}$ to the line of sight in unified models. Circles around data points indicate hot spots on the same side of the source as a distinct one-sided radio jet. 


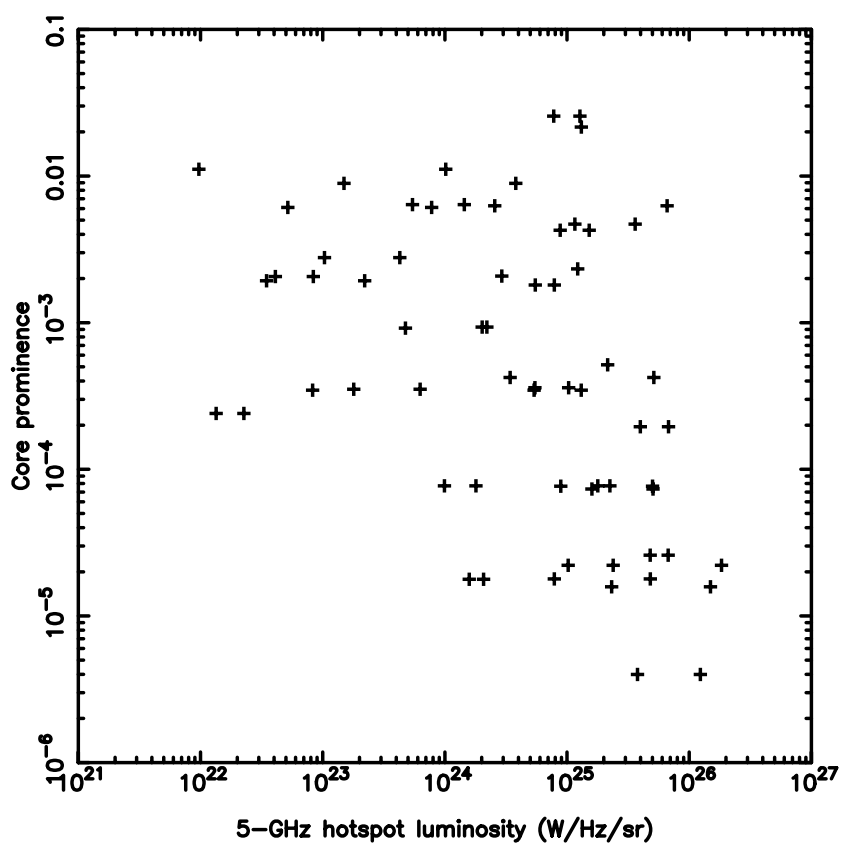

FIG. 8.-Core prominence plotted against hot spot luminosity for the X-ray hot spot sample.

apparent correlation of Figure 7 must be regarded as suggestive at best.

\section{DISCUSSION}

What models can account for these observations? We examine several in turn.

\subsection{SSC with Luminosity-dependent Departures from Equipartition}

One obvious possibility is that the high- $R$ values reflect a significant departure from equipartition; the belief that equipartition fields exist in hot spots is, after all, a result of the study of the most luminous hot spots (chosen because of their high predicted SSC flux densities). The departure from equipartition in terms of the ratio of equipartition to true magnetic field strengths, $B_{\mathrm{eq}} / B$, is approximately $R^{0.6}$, so that the magnetic field strength would have to be a factor of $\sim 100$ lower than the equipartition value in the hot spots with the highest $R$-values.

We can rule out a simple and attractive model in which such low magnetic fields account entirely for the luminosity- $R$ correlation. In this toy model, all hot spots have similar numbers of electrons and sizes. The radio luminosity $L_{R}$ goes as $B^{1+\alpha}$, and the IC luminosity scales in the same way (so long as the synchrotron photon field remains dominant). However, the equipartition prediction for SSC decreases more rapidly, since this depends on the equipartition estimate of electron density, which goes approximately as $L_{R}^{4 / 7}$ (Appendix B), as well as linearly on the observed photon density. Hence, we should find that $R \propto L^{-4 / 7}$, which is not far from the observed slope. We should also find that the hot spot radio luminosity scales approximately as $B_{\text {req, }}^{1.5}$, where $B_{\text {req }}$ is the magnetic field strength required to produce the observed X-ray emission by inverse Compton processes, and this is also just about consistent with the data for our sources. However, we would not expect to see the observed correlation between $178 \mathrm{MHz}$ total flux density and $R$ (Fig. 4) in this picture, unless the same ratio between the true and equipartition magnetic fields persisted throughout the source. Very low fields in lobes are incompatible with observations of lobe inverse Compton emission, among other things; we see high- $R$ hot spots in sources whose lobes clearly do not show the same ratio between the observed and predicted inverse Compton emission from CMB photon scattering (e.g., 3C 403; R. Kraft et al. 2004, in preparation).

More generally, there are several arguments that seem to us to disfavor an SSC model (to be accurate, SSC plus inverse Compton scattering of the $\mathrm{CMB}$ ) with a larger departure from equipartition in lower power sources:

1. We know (see $\S 1$ ) that some high- $R$ hot spots' X-ray spectra and/or spatial properties are inconsistent with a pure SSC model (the spectra of the best-studied high- $R$ sources are all found to be steep, $\alpha_{\mathrm{X}} \approx 1.0$ ).

2. In a few well-resolved cases (e.g., 3C 351; H02) the local value of $R$ would be even higher in places than the integrated value we quote, representing an even greater challenge for SSC.

3. The fact that a synchrotron model can be fitted through the radio, optical, and X-ray points in some high- $R$ sources would have to be a coincidence in an SSC model, although this is a weak constraint; the optical emission might also be SSC, as it is thought to be in a couple of low- $R$ sources (Hardcastle 2001; Brunetti 2002).

4. An SSC model cannot explain the effects of beaming, if these are real; beaming suppresses SSC emission, so that we should see a weak anticorrelation with proxies of beaming like core prominence, at least for hot spots on the jet side.

5. The special status of $R=1$ suggests that there are few hot spots with $B>B_{\text {eq }}$ : it is not obvious why the departures from equipartition should all be in the sense $B<B_{\text {eq }}$.

6. There is no obvious mechanism that fully explains the observed luminosity dependence of $R$.

\subsection{Luminosity-dependent Synchrotron Emission}

A synchrotron model is a good fit to the overall spectrum of some of the most extreme sources, such as 3C 390.3 and 3C 403; if we accept that X-ray synchrotron emission is possible in some hot spots, as it certainly is in the jets of FR I sources (e.g., Hardcastle et al. 2001b), then it may contribute to many of them. If the ability of a hot spot to produce X-ray synchrotron emission depended on its luminosity, then it might be the case that all hot spots have inverse Compton emission at a level consistent with $R=1$ and equipartition magnetic fields but that the low-luminosity hot spots have an additional synchrotron component that may greatly exceed the inverse Compton emission.

It has already been argued in studies of optical synchrotron hot spots (e.g., Meisenheimer et al. 1997; Brunetti et al. 2003) that the high-frequency break in the synchrotron spectrum is a function of hot spot luminosity, in the sense that optical emission is much commoner from hot spots of low radio luminosities. This fact can be explained (Brunetti et al. 2003) in terms of the lower synchrotron loss rates in the lower magnetic fields (assuming equipartition) and lower photon densities in hot spots of lower radio luminosity; we have already seen (Fig. 6) that there is a correlation between these quantities and $R$ in our objects. In a standard hot spot spatial/ spectral model (e.g., Heavens \& Meisenheimer 1987) the break in the synchrotron spectrum comes about when we average over both the acceleration region itself and the regions downstream of it, in which synchrotron and inverse Compton 
losses have had time to have an effect; we would not expect to see a break if we could resolve the acceleration region from the downstream emission. The high-frequency cutoff in the synchrotron spectrum is a direct indicator of physics in the acceleration region and results from inefficiency in particle acceleration at high energies: most importantly, from our point of view, particle acceleration will become inefficient if the energy loss timescale in the acceleration region (due to synchrotron and inverse Compton losses) becomes shorter than the acceleration timescale. The fact that essentially all our X-ray data points fall on or below the line of an extrapolation from the radio with $\alpha_{\mathrm{rx}}=1.0$ (§3) shows that any luminosity dependence of synchrotron radiation in the X-ray hot spots cannot simply be an effect of a changing frequency of the spectral break, as Brunetti et al. (2003) argued for optical hot spots; $\alpha=1.0$ is the canonical spectral index above the break, so that the break alone cannot produce the effect we see. This interpretation is supported by observations of well-studied luminous hot spots such as those of $3 \mathrm{C} \mathrm{405,} \mathrm{where} \mathrm{the} \mathrm{overall}$ spectrum requires the cutoff to be below the $\mathrm{X}$-ray region. We conclude that the luminosity dependence of $R$ must be an effect of the synchrotron cutoff.

A full calculation of the frequency of the cutoff $\nu_{c}$ depends on poorly known quantities such as the magnetic field strength in the acceleration region and the diffusion coefficient of relativistic particles. In the simplest case, with uniform magnetic field throughout the hot spot, a diffusion coefficient independent of both magnetic field and electron energy, and a nonrelativistic shock, it can be shown that $\nu_{c} \propto B /\left(\frac{2}{3} B^{2}+B_{\mathrm{IC}}^{2}\right)^{2}$, with the constant of proportionality depending on the numerical value of the diffusion coefficient, where $B$ is the magnetic field strength and $B_{\mathrm{IC}}$ is the equivalent inverse Compton field strength, defined as $B_{\mathrm{IC}}=\left(2 \mu_{0} U_{\mathrm{IC}}\right)^{1 / 2}$, with $U_{\mathrm{IC}}$ being the energy density in all photon fields. In this calculation $\nu_{c}$ does exhibit a change over the parameters of the hot spots we have studied that would be sufficient in magnitude to explain the observed effect, although the detailed correlation with $R$ is not particularly good (Fig. 9). Brunetti et al. (2003) consider two cases with less simplistic forms of the diffusion coefficient (Kolmogorov and Bohm diffusion coefficients) and are able to calculate corresponding numerical values for $\gamma_{\max }$ (their eq. [5]). The functional form of $\gamma_{\max }$ for the Bohm coefficient means that the cutoff frequency is essentially constant and (for the values quoted by Brunetti et al. 2003) lies well above the X-ray region for all our hot spots; the Kolmogorov coefficient gives a $\nu_{c}$ lying well below X-ray frequencies for equipartition magnetic field strengths in all the hot spots. This illustrates the strong dependence of the expectation on the unknown microphysics of the acceleration process. The basic principle of this model remains plausible: hot spot luminosity (and therefore magnetic field and photon energy density) is controlling the high-energy cutoff of the synchrotron spectrum.

Any relationship between $R$ and core prominence is not easy to explain in a model where much of the X-ray emission is synchrotron. $R$ does not have a simple dependence on beaming parameters in this model: the expected synchrotron flux of the hot spot increases with beaming, but so will the predicted inverse Compton flux density (from both SSC and CMB scattering), since the prediction we make is based on the $o b$ served radio flux density and takes no account of beaming. We calculated the expected variation of $R$ with angle to the line of sight $\theta$ for a source whose intrinsic (rest frame) properties were

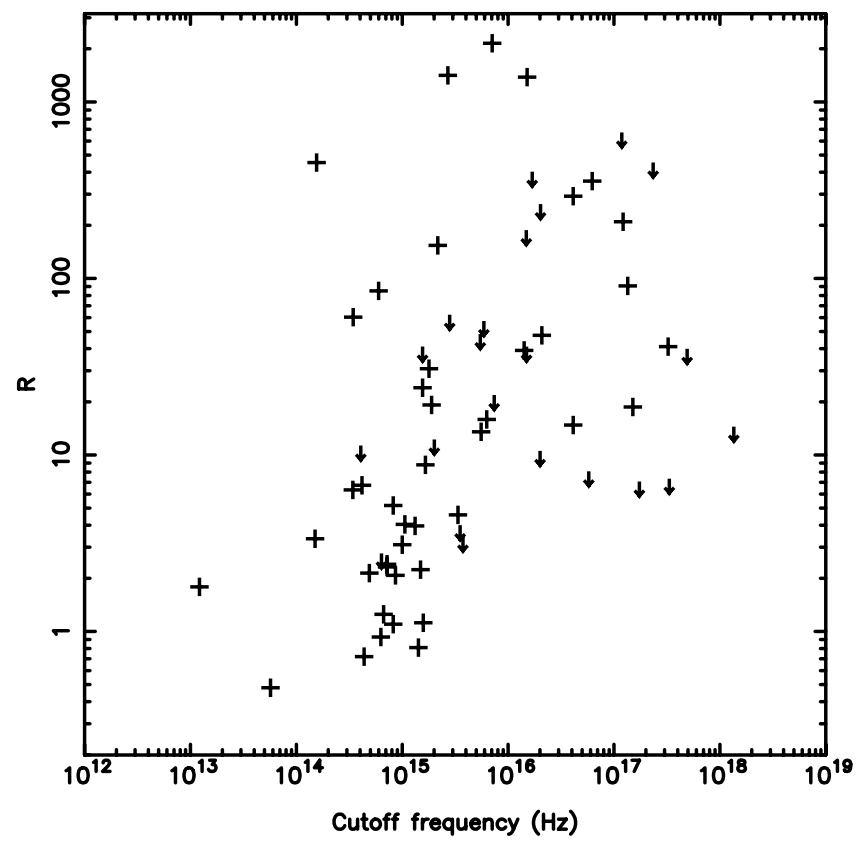

FIG. 9.- $R$ plotted against the synchrotron cutoff frequency for the hot spots in the sample, using the proportionality between $\nu_{c}$ and magnetic field strength for a constant diffusion coefficient quoted in the text. The constant of proportionality (i.e., the normalization of the $X$-axis) is chosen simply to illustrate that the magnitude of the effect could be significant in this situation and has no physical basis. The magnetic field strength used is the equipartition field derived from our models, and the photon energy density is a combination of the microwave background and the integrated synchrotron spectrum for each hot spot. A trend is apparent, in the sense that low- $R$ hot spots have low cutoffs while high- $R$ ones have high cutoffs, but the scatter is large.

held constant. For modest beaming factors, corresponding to $v / c \sim 0.3$, we find that $R$ does indeed increase as $\theta$ gets smaller, but only for extremely low luminosity hot spots, in which scattering of CMB photons is the dominant IC process; for more luminous hot spots the trend is reversed, and we would expect $R$ to be largest for hot spots that are beamed away from us (that is, on the counterjet side of beamed sources). In any case, the amount of variation introduced by this process into the $R$-value distribution is small, no more than a factor of 2 between minimum and maximum values, for $v / c \sim 0.3$. Much higher speeds $(v / c \gtrsim 0.9)$ would be required, for reasonable hot spot luminosities, to obtain the order-of-magnitude scatter in the $R$ parameter (after accounting for the luminosity dependence) that appears to be present in Figure 5.

\subsection{Deceleration and Beaming}

We have already shown $(\S 3)$ that the apparent relationship between proxies of beaming, such as core prominence, and the X-ray brightness of the hot spot (H02; Georganopoulos \& Kazanas 2003) may at least partly be a selection effect in the available X-ray data. The current sample of X-ray hot spots, at least at low hot spot luminosities, is strongly biased toward beamed objects, while beamed objects are known to have brighter, more compact, flatter spectrum hot spots on the jet side. It is now known that there are narrow-line radio sources that should lie close to the plane of the sky that have high $R$ and a few examples of sources (for example, 3C 228 and 3C 321) where hot spots on both sides of the source have high $R$. It is not clear, therefore, whether there is any beaming effect that needs to be explained from an X-ray perspective. 
If there is any beaming effect, then the two models discussed so far both have difficulty in explaining it, so alternative models must be considered. The standard way of explaining the (effectively) high- $R$ values in the X-ray jets of coredominated quasars is to invoke highly relativistic bulk speeds and the consequent boost of the energy density of the CMB in the rest frame of the jet. We regard this model as untenable in the present case, for several reasons. First, large bulk Lorentz factors are required for even moderate $R$-values (see the discussion of $3 \mathrm{C} 351$ in $\mathrm{H} 02$ ), and these in turn constrain the source to lie at a small angle to the line of sight; this cannot possibly be the case for all or even most of our high- $R$ objects, which are drawn from a low-frequency-selected, lobedominated sample and include objects that, in unified models, must be close to the plane of the sky. Secondly, in the standard picture, the radio emission from the hot spots comes from the postshock region, and so high bulk Lorentz factors are hard to achieve; although there are some effects that are best explained by moderate relativistic beaming in the postshock flow, bulk Lorentz factors of $\sim 10$ have never been required by observation and are in fact inconsistent with the known properties of hot spots.

A more viable model involving beaming effects is that of Georganopoulos \& Kazanas (2003; see also $\S 1$ ). The picture they describe almost certainly has to be true at some level, but a quantitative test is difficult, since it relies on knowledge of the velocity and electron density structure of the jet upstream of the hot spot that is hard to obtain observationally. In addition, this model cannot account for all the features of our data, such as the hot spot luminosity dependence of $R$. However, our data clearly do not rule out a beaming effect at some level, and, if it is present, the standard inverse Compton or synchrotron models cannot account for it without involving large speeds. A full test of this type of beaming model must await an unbiased sample of hot spots in which orientation and luminosity effects can be clearly separated.

\section{HOT SPOTS AND JET KNOTS}

As discussed in $\S 1$, we have tried to distinguish between hot spots, defined as structures where the well-collimated flow of the jet terminates, and jet knots, where the assumption is that the jet continues more or less unaffected by whatever process produces the increase in synchrotron emissivity. The key physical differences between the two systems are (1) that there is probably not a strong shock in FR II jet knots, since there is little evidence that the jets decelerate there; and (2) that the particles in jet knots probably have a shorter dwell time in the region of interest, since the downstream flow speed is likely to be faster, which could lead to spectral differences even if the acceleration processes are similar. In practice the distinction between the two types of feature is difficult to draw observationally: there are several features that we have considered to be hot spots in our sample (e.g., 3C $390.3 \mathrm{~N}, 3 \mathrm{C}$ $403 \mathrm{~F} 6,3 \mathrm{C} 275.1 \mathrm{~N}$ ) that might well be jet knots in which the continuing jet is poorly defined. Equally, it must be the case that there is continued collimated flow out of primary hot spots in cases in which there is optical or possible X-ray synchrotron emission in the secondary hot spot, requiring in situ particle acceleration: $3 \mathrm{C}$ 351's hot spots $\mathrm{J}$ and $\mathrm{K}$ are a good example. We see no observational differences between these borderline jet knot/hot spot sources and clearly defined terminal hot spots.
Should we therefore try to apply the results of the present work to jet knots as well as to hot spots? As we have argued above ( $\S 4.3$ ), the generally favored jet X-ray emission mechanism for core-dominated quasars cannot apply to more than a small subset of our sources, and particularly not the narrowline objects, some of which exhibit either possible jet-related $\mathrm{X}$-ray knots (e.g., 3C 403) or clear jet-related X-ray emission (e.g., 3C 321 and 3C 452 in Appendix A). The X-ray emission mechanism here seems likely to be synchrotron, as in the jets of low-luminosity FR I sources. A full analysis of the known FR II jet-related X-ray emission is beyond the scope of the present paper, but from our work on hot spots we can make the "prediction" (borne out by the observations that we are currently aware of) that jet-related X-ray synchrotron emission in FR II sources will be seen mostly in low-luminosity jet knots and therefore should be particularly easy to find in low-luminosity FR II sources. The hot spot behavior is also qualitatively similar to what is seen in the jets of some powerful quasars, such as 3C 273 (Sambruna et al. 2001; Marshall et al. 2001), in which the X-ray-toradio ratio of jet knots decreases as the knot radio flux density increases.

\section{CONCLUSIONS}

We have shown that the properties of the X-ray emission of hot spots depend strongly on their overall radio luminosity. High-luminosity hot spots, of the type originally examined in inverse Compton studies, consistently show X-ray emission that is close to being consistent with the predictions of an SSC model with an equipartition magnetic field. Low-luminosity hot spots sometimes (and maybe always) have X-ray emission that is much brighter than would be expected in this model. We argue the following:

1. The good agreement between IC models and data seen for the luminous hot spots continues to suggest that the X-ray emission mechanism in these systems really is SSC, that magnetic fields really are in equipartition, and that populations of protons that dominate energetically by large factors $(\gtrsim 100)$ and/or very small filling factors are not present.

2. Models in which the unexpectedly strong X-ray emission from some low-luminosity hot spots indicates a large departure from equipartition in these objects are not plausible for a number of reasons; synchrotron emission is more likely.

3. If a synchrotron model is adopted, the high-frequency cutoff of the synchrotron spectrum must be dependent on luminosity in order to explain the X-ray emission from all hot spots. This is physically plausible, but a fully quantitative test depends on the microphysics of the acceleration process.

4. There is little significant evidence that relativistic beaming is important in the current sample; an unbiased sample of X-ray hot spots would be of great importance in testing beaming models.

5. It may be possible to extend our conclusions on hot spots to the jet-related X-ray features seen in a number of FR II sources, particularly those at relatively large angles to the line of sight; if so, we would expect that they would show the same luminosity dependence, in the sense that only low-luminosity jets would show strong X-ray synchrotron emission. A synchrotron origin for the jets in these sources would suggest a continuity between their properties and those of the lower power FR I sources, for which a synchrotron interpretation is well established (e.g., Hardcastle et al. 2001b). 
M. J. H. thanks the Royal Society for a research fellowship. This work was partially supported by NASA grant GO3$4132 \mathrm{X}$. We thank C. Cheung and an anonymous referee for helpful comments on the manuscript. We would like to thank those involved in setting up full online access to the VLA archive, without which this project would have been impossible, and in particular John Benson for quick assistance with problems that arose with the online archive in the early stages of its operation. We thank Rick Perley for supplying images of the hot spots of Pictor A. The National Radio Astronomy Observatory is a facility of the National Science Foundation operated under cooperative agreement by Associated Universities, Inc.

\section{APPENDIX A}

\section{NEWLY DETECTED HOT SPOTS}

Below we present images of the hot spots newly detected in the course of this work that are not expected to be discussed in more detail in other papers.

\section{A1. 3C 6.1}

The northern hot spot of this narrow-line source is the clearest detection (Fig. 10), but there is a weak detection of the southern hot spot too, at well over $3 \sigma$ significance. Some X-ray emission is associated with the lobes.

\section{A2. $3 \mathrm{C} 47$}

This quasar's bright southern hot spot (on the jet side) is detected (Fig. 11), but there is no obvious detection of the fainter northern hot spot. Extended emission is clearly visible in the X-ray image: since it is extended in the direction of the lobes, some of it may well be inverse Compton emission, but probably a large fraction of it comes from a cluster environment, particularly as 3C 47 exhibits a strong Laing-Garrington effect (e.g., Leahy 1996).

\section{A3. 3C 109}

The southern hot spot of this broad-line radio galaxy is detected (Fig. 12); the southern side is the jet side, and a weak jet can be traced into the hot spot (Gilbert et al. 2004). Some excess extended emission from the lobes can be seen in these images and is consistent with inverse Compton emission at approximately the level expected from equipartition in the lobes.

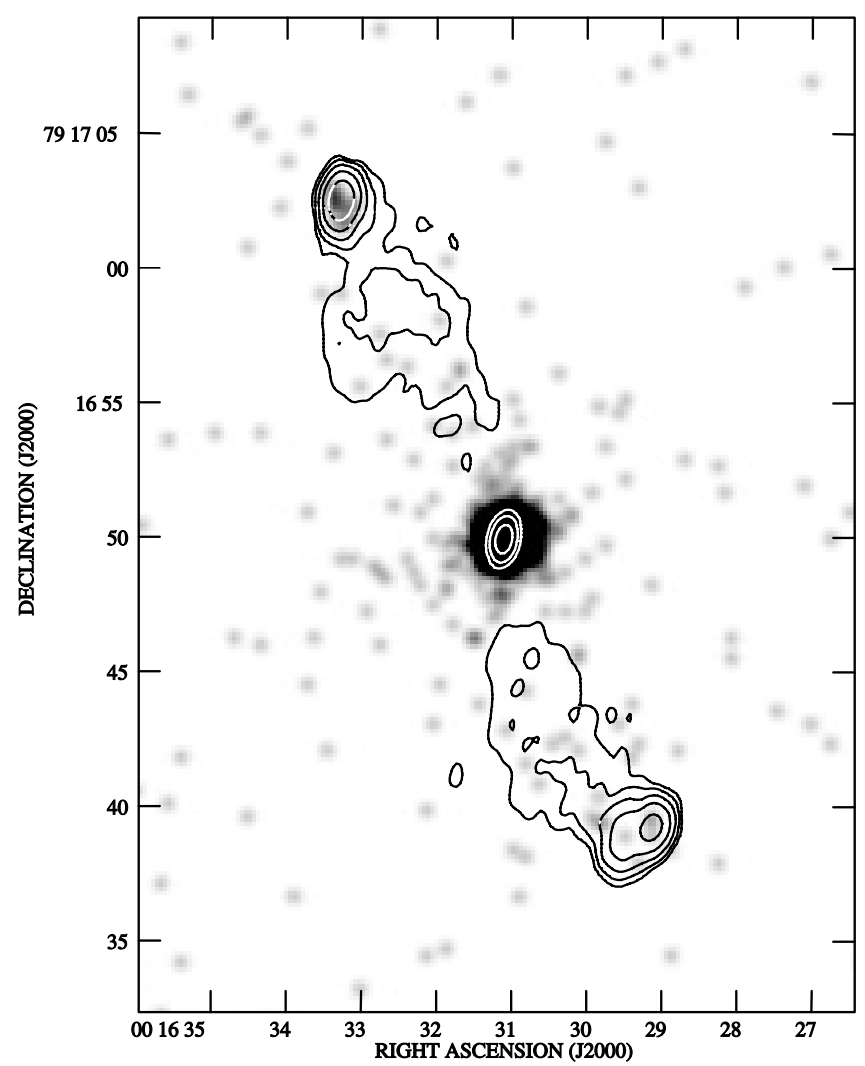

Fig. 10.-X-ray hot spots of $3 \mathrm{C} 6.1$. The gray scale shows the $0.5-5 \mathrm{keV}$ Chandra counts smoothed with a 0 ". 5 FWHM Gaussian; black corresponds to 1 count per 0.246 pixel. The contours are of the $8.4 \mathrm{GHz}$ B configuration VLA map at $0^{\prime \prime} 94 \times 0$ "' 53 resolution and are at $0.2 \times(1,4,16, \ldots) \mathrm{mJy}^{\prime}$ beam ${ }^{-1}$. 


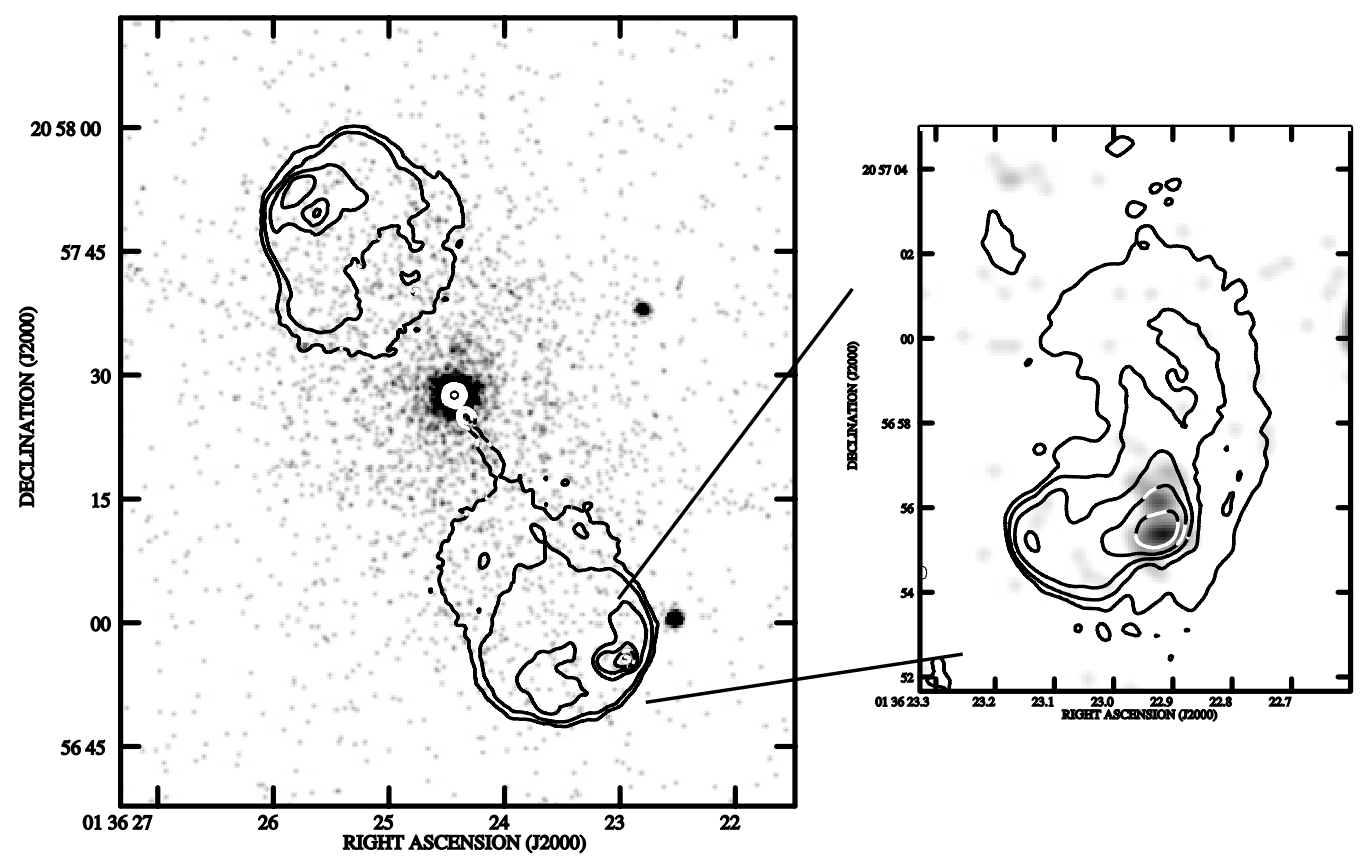

FIG. 11.-Southern X-ray hot spot of $3 \mathrm{C} 47$. The main gray scale (left) shows the $0.5-5 \mathrm{keV}$ Chandra counts smoothed with a 0." 5 FWHM Gaussian; black corresponds to 5 counts. The contours are of a $1^{\prime \prime} .0$ resolution $1.6 \mathrm{GHz}$ VLA map taken from Leahy et al. $(1998)$ and are at $0.3 \times(1,4,16, \ldots) \mathrm{mJy} \mathrm{beam}^{-1}$. The inset $(r i g h t)$ is the same X-ray image with the same gray-scale level, but with contours from the $0.39 \times 0$ "' 36 resolution $4.8 \mathrm{GHz}$ VLA map at $80 \times(1,4,16, \ldots) \mu \mathrm{Jy} \mathrm{beam}^{-1}$.

\section{A4. 3C 173.1}

There is a weak detection of the southern hot spot of this low-excitation radio galaxy (Fig. 13), at well over $3 \sigma$ significance. The extended emission here is again probably a combination of inverse Compton emission and a thermal environment. The hot spot here is on the counterjet side.

\section{A5. 3C 321}

Both hot spots of this nearby narrow-line FR II source are detected, weakly but convincingly (Fig. 14). In addition, there is X-ray emission from a weak radio jet entering the southern hot spot from slightly west of north (just visible on our high-resolution contour

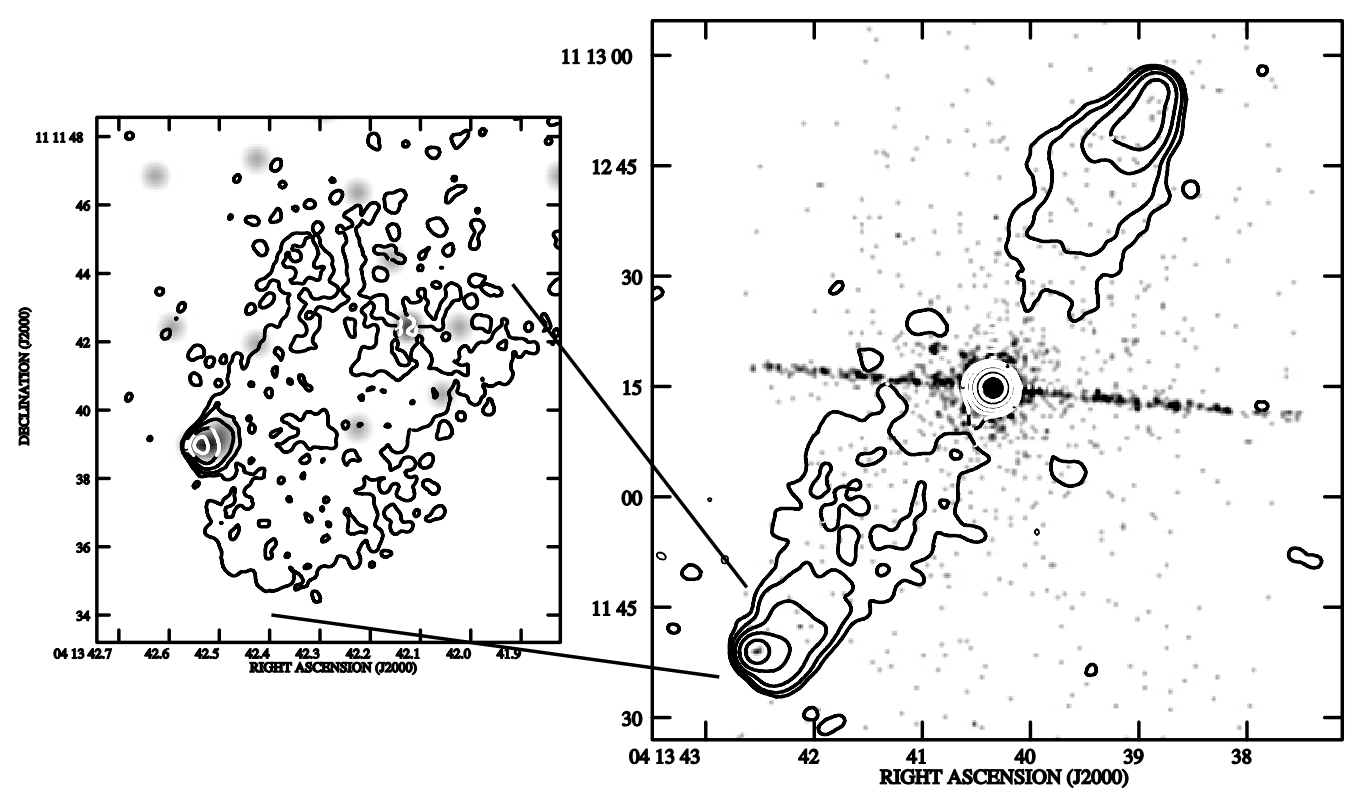

FIG. 12.- Southern X-ray hot spot of 3C 109. The main gray scale (right) shows the 0.5-5 keV Chandra counts smoothed with a 0."5 FWHM Gaussian; black

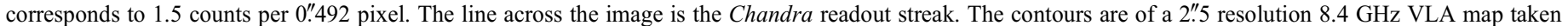

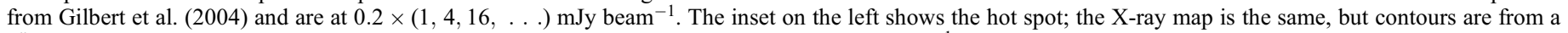
0 ".25 resolution $8.4 \mathrm{GHz}$ map also from Gilbert et al. $(2004)$, at $0.1 \times(1,4,16, \ldots) \mathrm{mJy} \mathrm{beam}^{-1}$. 


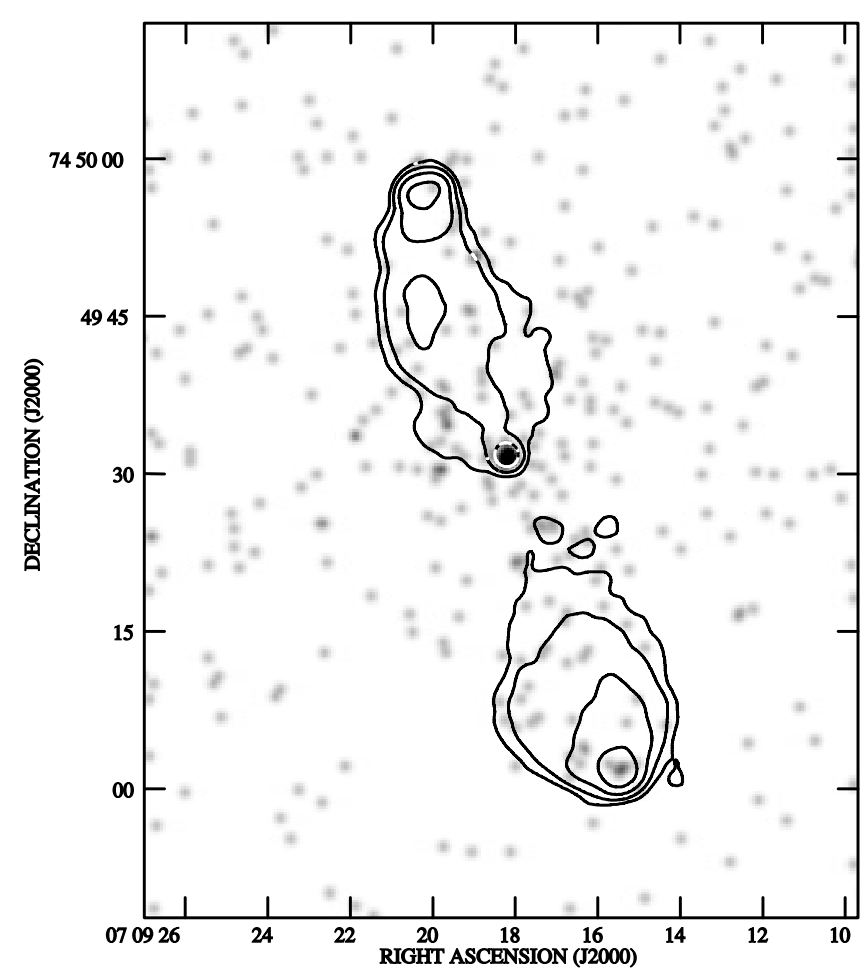

FIG. 13.- Southern X-ray hot spot of $3 \mathrm{C} 173.1$. The gray scale shows the $0.5-5 \mathrm{keV}$ Chandra counts smoothed with a 1".0 FWHM Gaussian; black corresponds to 4 counts per $00^{\prime \prime} 246$ pixel. The contours are of a 1 ."7 resolution $8.4 \mathrm{GHz}$ VLA map taken from Hardcastle et al. $(1997)$ and are at $0.2 \times(1,4,16, \ldots) \mathrm{mJy}$ beam ${ }^{-1}$.

map). The nuclear region shows very unusual structure. The component associated with the radio core is extended and, if the relative astrometry of the Chandra and radio data is correct, shows quite strong X-ray emission from the radio-weak jet pointing southeast; the northwest compact bright X-ray source is positionally coincident not with the bright northwest radio jet, which is not clearly detected in X-rays, but with the nearby companion galaxy seen with HST (Martel et al. 1999) if we align the center of the host galaxy with the radio core and the brightest component in the X-ray (the HST data have the usual arcsecond-scale astrometric offset, and there are no obvious independent features with which to align the two data sets). There is also extended emission around the two galaxies that appears to be spatially coincident with the known optical line-emitting material (Baum et al. 1988). The strongly different jetcounterjet asymmetry in the radio and X-ray is hard to explain in a model in which the jet and counterjet are intrinsically symmetrical.

\section{A6. 3C 324}

Both hot spots of this small narrow-line radio galaxy are detected (Fig. 15), the eastern hot spot clearly, the western one more marginally.

\section{A7. 3C 452}

The western hot spot of this low-redshift narrow-line radio galaxy is clearly detected (Fig. 16), as is a faint linear X-ray feature pointing west from the nucleus toward the known radio jet in the western lobe (although the features shown in Fig. 16 have no detected radio counterparts on high-resolution maps). Extended emission associated with the lobes has already been reported and is modeled in terms of inverse Compton emission (Isobe et al. 2002).

\section{APPENDIX B}

\section{SYNCHROTRON AND INVERSE COMPTON EMISSION}

To guide the reader in interpreting the physics of synchrotron and inverse Compton emission, we include here a brief sketch of the underlying physics. In practice these calculations are carried out by the computer code discussed in the paper and in Hardcastle et al. (1998), but it is useful to set out the theoretical underpinning of the code's results and to outline the key dependencies of the model parameters.

We take as a fiducial assumption (which can then be tested by observation) the equipartition of energy between electrons (or more generally particles of all kinds) and magnetic field. If the electron energy spectrum (number per unit energy per unit volume) is described by a function $N(E)$, then equipartition implies (in SI units)

$$
\frac{B^{2}}{2 \mu_{0}}=\int_{E_{\min }}^{E_{\max }} E N(E) d E+u_{\mathrm{NR}},
$$




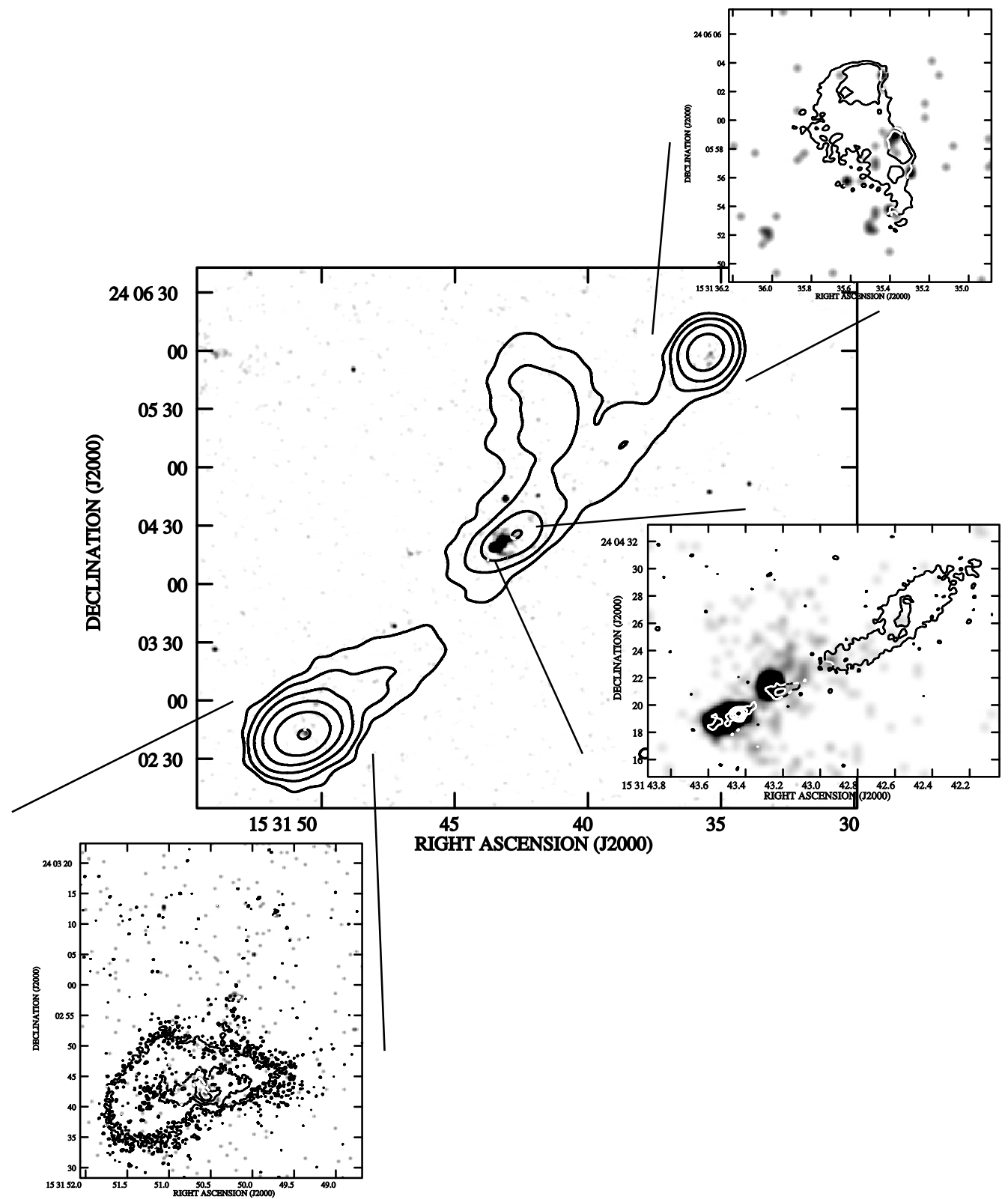

Fig. 14. - X-ray hot spots of 3C 321. The main gray scale shows the $0.5-5 \mathrm{keV}$ Chandra counts smoothed with a 2".0 FWHM Gaussian; black corresponds to 1 count per 0.492 pixel. The contours are of a $1.4 \mathrm{GHz}$ VLA map with $15^{\prime \prime} \times 13^{\prime \prime}$ resolution and are at $2 \times(1,4,16, \ldots) \mathrm{mJy}^{\prime}$ beam ${ }^{-1}$. Insets show the same map smoothed with a 0 " 5 FWHM Gaussian, and contours from a $4.8 \mathrm{GHz}$ VLA map with $0.45 \times 0$ " 40 resolution, at $0.15 \times(1,4,16, \ldots) \mathrm{mJy} \mathrm{beam}^{-1}$; black is 1 count pixel $^{-1}$ for the hot spots and 5 counts pixel ${ }^{-1}$ for the nuclear inset.

where $E_{\min }$ and $E_{\max }$ give the range of electron energies, $B$ is the magnetic field strength, $\mu_{0}$ is the permeability of free space, and $u_{\mathrm{NR}}$ is the energy density in nonradiating particles. It is conventional to let $\kappa$ be the ratio of the energy densities in nonradiating and radiating particles: then

$$
\frac{B^{2}}{2 \mu_{0}}=(1+\kappa) \int_{E_{\min }}^{E_{\max }} E N(E) d E
$$

It is easy to see that the $(1+\kappa)$ term can also be used to describe an arbitrary departure from equipartition between the electrons and magnetic field. Our fiducial assumption is equivalent to $\kappa=0$.

Now let us consider for simplicity a power-law distribution of electron energies, $N(E) d E=N_{0} E^{-p} d E$. Then the integral can be carried out analytically:

$$
\frac{B^{2}}{2 \mu_{0}}=(1+\kappa) N_{0} I,
$$




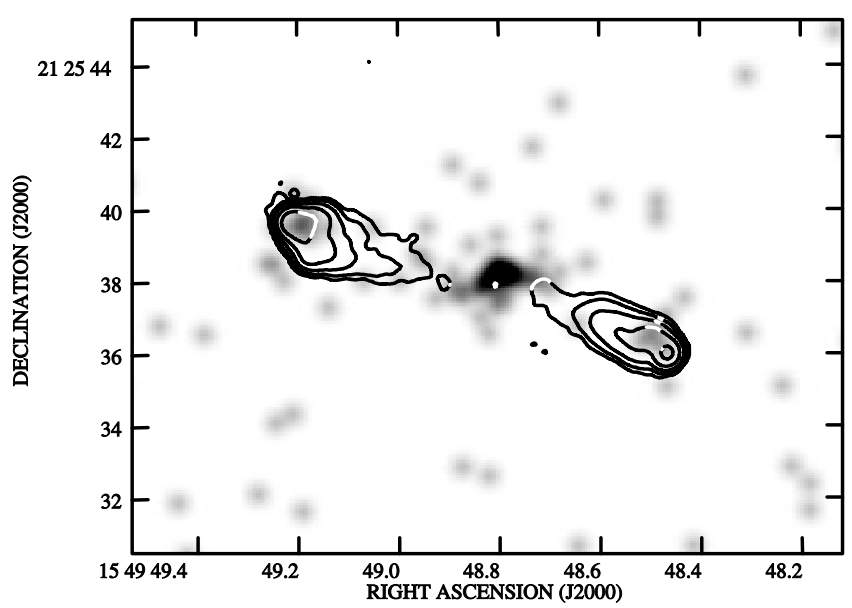

FIG. 15.-X-ray hot spots of 3C 324. The gray scale shows the $0.5-5 \mathrm{keV}$ Chandra counts smoothed with a 0".5 FWHM Gaussian; black corresponds to 4 counts per 0 ".246 pixel. The contours are of the $4.8 \mathrm{GHz}$ VLA A configuration map at 0.39 resolution and are at $0.15 \times(1,4,16, \ldots) \mathrm{mJy}^{\prime \prime}$ beam ${ }^{-1}$.

where

$$
I= \begin{cases}\ln \left(E_{\max } / E_{\min }\right), & p=2, \\ \frac{1}{2-p\left[E_{\max }^{(2-p)}-E_{\min }^{(2-p)}\right]}, & p \neq 2 .\end{cases}
$$

In practice, as described in the text, we may use more complicated electron energy spectra, and then it is easiest to determine $I$ numerically.

The volume synchrotron emissivity of the ensemble of electrons at a given source-frame frequency $\nu$ may be written (e.g., Longair 1994)

$$
J(\nu)=\frac{\sqrt{3} B e^{3} \sin \theta}{4 \pi \epsilon_{0} c m_{e}} \int_{E_{\min }}^{E_{\max }} F(x) N(E) d E .
$$

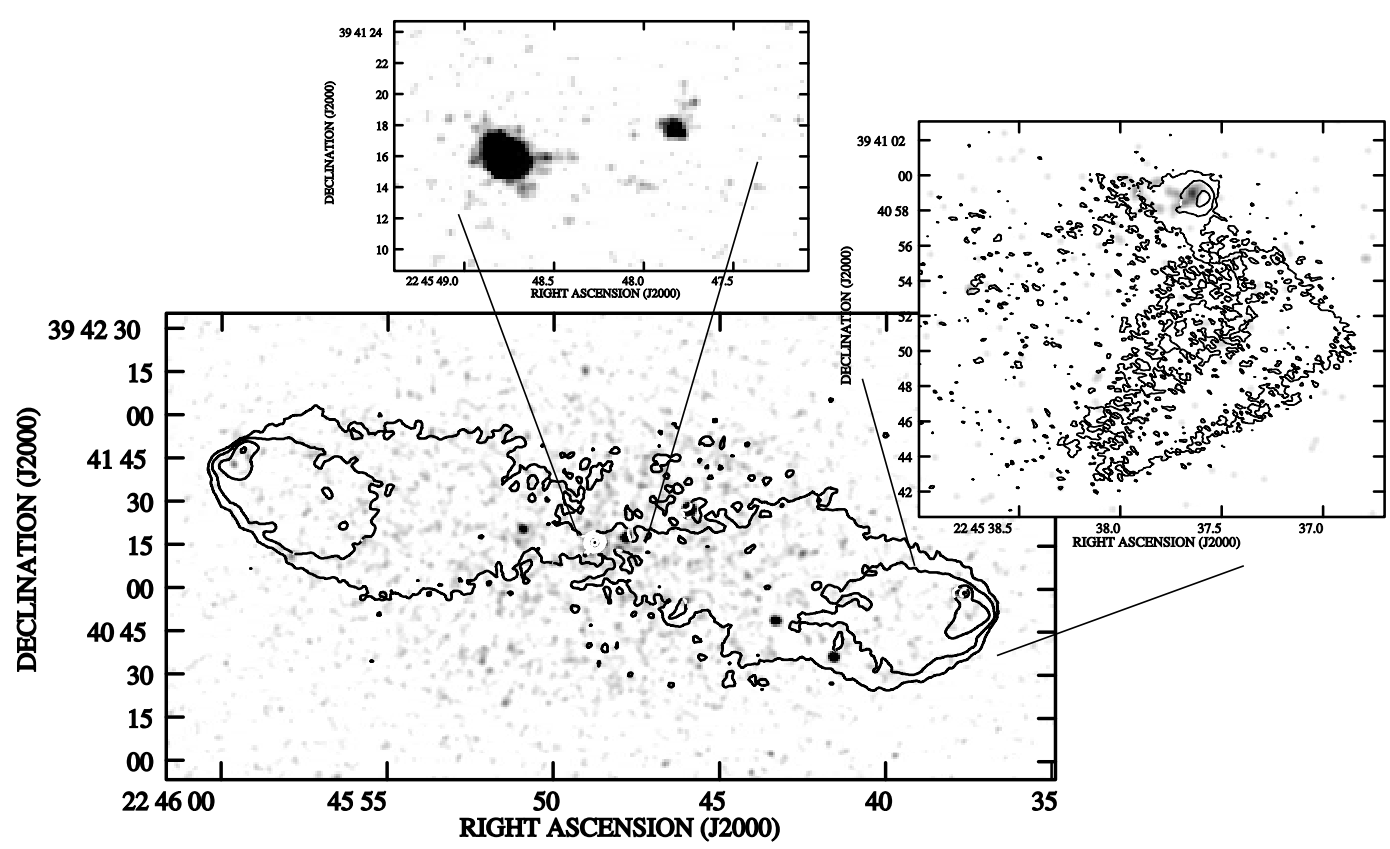

Fig. 16.-Western X-ray hot spot of 3C 452. The main gray scale shows the $0.5-5 \mathrm{keV}$ Chandra counts smoothed with a 2".0 FWHM Gaussian; black corresponds to 10 counts per 0.246 pixel. The contours are of an $8.4 \mathrm{GHz}$ VLA map with 2.5 resolution from Black et al. $(1992)$ and are at $0.3 \times(1,4,16, \ldots)$ mJy beam $^{-1}$. Insets show the same X-ray image smoothed with a 0 ". 5 FWHM Gaussian, with black being 1 count per 0 "'246 pixel. Right: Hot spot: contours are of an $8.4 \mathrm{GHz}$ VLA map with $0{ }^{\prime \prime} 25$ resolution from Black et al. $(1992)$ and are at $0.1 \times(1,4,16, \ldots)$ mJy beam ${ }^{-1}$. Top: Inner jet (no contours are shown). 
Here $m_{e}$ is the mass of the electron, $e$ is its charge, and $c$ is the speed of light; $\epsilon_{0}$ is the permittivity of free space. In addition, $\theta$ is the pitch angle of the electrons with respect to the magnetic field direction, and $x$ is defined by

$$
x=\frac{4 \pi m^{3} c^{4}}{3 e} \frac{\nu}{E^{2} B \sin \theta} .
$$

$F(x)$ is a sharply peaked function of $x$, reflecting the fact that electrons of a given energy radiate at a well-defined frequency:

$$
F(x)=x \int_{x}^{\infty} K_{5 / 3}(z) d z
$$

where $K_{5 / 3}$ is the modified Bessel function of order 5/3. Assuming pitch-angle isotropy, we can integrate equation (B4) over pitch angle and our assumed electron power law (e.g., Longair 1994) to find that

$$
J(\nu)=C N_{0} \nu^{-(p-1) / 2} B^{(p+1) / 2},
$$

where

$$
C=c(p) \frac{e^{3}}{\epsilon_{0} c m_{e}}\left(\frac{m_{e}^{3} c^{4}}{e}\right)^{-(p-1) / 2}
$$

and $c(p)$ is of order 0.05 and depends only weakly on $p$. Since equation (B6) describes a power law in frequency, the electron energy index $p$ can be determined by observation: typical values lie in the range $2-3$, and our assumption of a low-frequency spectral index $\alpha=0.5$ corresponds to $p=2$.

If we know the emissivity $J$, we can use equation (B6) to eliminate $N_{0}$ from equation (B2),

$$
\frac{B^{2}}{2 \mu_{0}}=(1+\kappa) \frac{J(\nu)}{C} \nu^{(p-1) / 2} B^{-(p+1) / 2} I
$$

and we can now solve for $B$ :

$$
B=\left[2 \mu_{0}(1+\kappa) \frac{J(\nu)}{C} \nu^{(p-1) / 2} I\right]^{2 /(p+5)} .
$$

In practice, we take account of the fact that $J(\nu)$ is not always a power law by numerically integrating equation (B4) and then solving equation (B7) numerically with a root-finding algorithm, but the main dependencies are encapsulated in equation (B8). Since the energy density is proportional to $B^{2}$, we can see that it increases as $(1+\kappa)^{4 / p+5}$; thus, a nonzero value of $\kappa$ affects the magnetic field strength in the expected sense. Moreover, we can now substitute back into equation (B6) to eliminate $B$ : this gives

$$
J(\nu)^{4 /(p+5)}=C^{4 /(p+5)} N_{0} \nu^{-[2(p+1) /(p+5)]}\left[2 \mu_{0}(1+\kappa) I\right]^{(p+1) /(p+5)},
$$

and, since $J(\nu)$ and $\nu$ are known and constant for a given observation and a known source geometry, we can see that the number density of electrons is expected to decrease with increasing $\kappa$ :

$$
N_{0} \propto(1+\kappa)^{-(p+1) /(p+5)} .
$$

If the geometry is doubtful, the calculated emissivity is a function of volume: $J \propto S / V$, where $S$ is the observed flux density. Thus, we expect

$$
N_{0} \propto V^{-4 /(p+5)}
$$

For sphere of radius $r$ with uniform particle and magnetic field density, the SSC emissivity at a given frequency $\nu_{1}$ is given by (Hardcastle et al. 1998)

$$
J_{\mathrm{IC}}\left(\nu_{1}\right)=\frac{9}{16} m_{e}^{2} c^{4} \nu_{1} \sigma_{\mathrm{T}} r \int_{E_{\min }}^{E_{\max }} \int_{\nu_{\min }}^{\nu_{\max }} \frac{N(E) J\left(\nu_{0}\right)}{E^{2} \nu_{0}^{2}} f(x) d \nu_{0} d E
$$

where $m_{e}$ is the electron mass, $c$ is the speed of light, $\sigma_{\mathrm{T}}$ is the Thomson cross section, $J\left(\nu_{0}\right)$ is the synchrotron emissivity as a function of frequency, and $f(x)$ is a function of $E, \nu_{1}$, and $\nu_{0}$ defined by Rybicki \& Lightman (1979). The code we use performs this integration numerically for a given synchrotron and electron energy spectrum and also carries out the similar calculation for illumination from the microwave background radiation; an analytical form of the integral for power-law electron and photon distributions could be derived but is not necessary here. The key feature of this equation is that the inverse Compton emissivity (which determines the predicted IC flux density and thus $R$ for a given observed flux) is linear in the number density of electrons 
$N(E)$ and thus linear in $N_{0}$ for the power-law analysis we have described above. For a given source, with known spatial and spectral properties, it is the dependence of $N_{0}$ on $(1+\kappa)$ given by equation (B10) that primarily determines the value of $R$. [If the spectrum is not a pure power law, the change in the form of $J\left(\nu_{0}\right)$ as a result of the change in $B$ also has a nonnegligible effect.]

If the volume is not known, for example, because of a low filling factor, then the dependence of $J_{\text {IC }}$ on volume can be determined from above:

$$
J_{\mathrm{IC}} \propto V^{1 / 3} V^{-4 /(p+5)} V^{-1}=V^{-2 / 3-4 /(p+5)},
$$

and this means that the observed inverse Compton flux density, which is proportional to $V J_{\mathrm{IC}}$, goes as $V^{1 / 3-4 /(p+5)}$. This is a very weak dependence for plausible $p$-values: for $p=2, S_{\mathrm{IC}} \propto V^{-5 / 21}$.

\section{REFERENCES}

Akritas, M. G., \& Siebert, J. 1996, MNRAS, 278, 919

Baars, J. W. M., Genzel, R., Pauliny-Toth, I. I. K., \& Witzel, A. 1977, A\&A, 61,99

Baum, S. A., Heckman, T., Bridle, A., van Breugel, W., \& Miley, G. 1988, ApJS, 68, 643

Belsole, E., Worrall, D. M., Hardcastle, M. J., Birkinshaw, M., \& Lawrence, C. R. 2004, MNRAS, in press

Black, A. R. S., Baum, S. A., Leahy, J. P., Perley, R. A., Riley, J. M., \& Scheuer, P. A. G. 1992, MNRAS, 256, 186

Bondi, M., Brunetti, G., Comastri, A., \& Setti, G. 2003, NewA Rev., 47, 443

Bridle, A. H., Hough, D. H., Lonsdale, C. J., Burns, J. O., \& Laing, R. A. 1994, AJ, 108, 766

Bridle, A. H., \& Perley, R. A. 1984, ARA\&A, 22, 319

Brunetti, G. 2002, in ASP Conf. Ser. 250, Particles and Fields in Radio Galaxies, ed. R. A. Laing \& K. M. Blundell (San Francisco: ASP), 238

Brunetti, G., Bondi, M., Comastri, A., Pedani, M., Varano, S., Setti, G., \& Hardcastle, M. J. 2001, ApJ, 561, L157

Brunetti, G., Bondi, M., Comastri, A., \& Setti, G. 2002, A\&A, 381, 795

Brunetti, G., Mack, K. H., Prieto, M. A., \& Varano, S. 2003, MNRAS, 345, L40

Crawford, C. S., \& Fabian, A. C. 2003, MNRAS, 339, 1163

Donahue, M., Daly, R. A., \& Horner, D. J. 2003, ApJ, 584, 643

Fabian, A. C., Sanders, J. S., Crawford, C. S., \& Ettori, S. 2003, MNRAS, 341, 729

Fernini, I., Burns, J. O., Bridle, A. H., \& Perley, R. A. 1993, AJ, 105, 1690

Georganopoulos, M., \& Kazanas, D. 2003, ApJ, 589, L5

Gilbert, G., Riley, J. M., Hardcastle, M. J., Croston, J. H., Pooley, G. G., \& Alexander, P. 2004, MNRAS, 351, 845

Hardcastle, M. J. 2001, A\&A, 373, 881

Hardcastle, M. J., Alexander, P., Pooley, G. G., \& Riley, J. M. 1997, MNRAS, 288,859 1999, MNRAS, 304, 135

Hardcastle, M. J., Birkinshaw, M., Cameron, R., Harris, D. E., Looney, L. W., \& Worrall, D. M. 2002, ApJ, 581, 948 (H02)

Hardcastle, M. J., Birkinshaw, M., \& Worrall, D. M. 1998, MNRAS, 294, 615 2001a, MNRAS, 323, L17 2001b, MNRAS, 326, 1499

Hargrave, P. J., \& Ryle, M. J. 1974, MNRAS, 166, 305

Harris, D. E., Carilli, C. L., \& Perley, R. A. 1994, Nature, 367, 713

Harris, D. E., Leighly, K. M., \& Leahy, J. P. 1998, ApJ, 499, L149

Harris, D. E., et al. 2000, ApJ, 530, L81

Heavens, A. F., \& Meisenheimer, K. 1987, MNRAS, 225, 335

Isobe, N., Tashiro, M., Makishima, K., Iyomoto, N., Suzuki, M., Murakami, M. M., Mori, M., \& Abe, K. 2002, ApJ, 580, L111
Kapahi, V. K., \& Murphy, D. W. 1990, in Parsec-scale Radio Jets, ed. J. A. Zensus \& T. J. Pearson (Cambridge: Cambridge Univ. Press), 313

Kataoka, J., Edwards, P., Georganopoulos, M., Takahara, F., \& Wagner, S. 2003, A\&A, 399, 91

Kronberg, P. P. 1986, Canadian J. Phys., 64, 449

Laing, R. A. 1982, in IAU Symp. 97, Extragalactic Radio Sources, ed. D. S. Heeschen \& C. M. Wade (Dordrecht: Reidel), 161

Laing, R. A., Riley, J. M., \& Longair, M. S. 1983, MNRAS, 204, 151

Leahy, J. P. 1996, Vistas Astron., 40, 173

Leahy, J. P., Black, A. R. S., Dennett-Thorpe, J., Hardcastle, M. J., Komissarov, S., Perley, R. A., Riley, J. M., \& Scheuer, P. A. G. 1997, MNRAS, 291, 20

Leahy, J. P., Bridle, A. H., \& Strom, R. G. 1998, An Atlas of DRAGNs, http:// www.jb.man.ac.uk/atlas

Longair, M. S. 1992, High Energy Astrophysics, Vol. 1 (2nd ed.; Cambridge: Cambridge Univ. Press)

- 1994, High Energy Astrophysics, Vol. 2 (2nd ed.; Cambridge: Cambridge Univ. Press)

Marshall, H. L., et al. 2001, ApJ, 549, L167

Martel, A. R., et al. 1999, ApJS, 122, 81

Meisenheimer, K., Röser, H.-J., Hiltner, P. R., Yates, M. G., Longair, M. S., Chini, R., \& Perley, R. A. 1989, A\&A, 219, 63

Meisenheimer, K., Yates, M. G., \& Röser, H.-J. 1997, A\&A, 325, 57

Morganti, R., Oosterloo, T. A., Reynolds, J. E., Tadhunter, C. N., \& Migenes, V. 1997, MNRAS, 284, 541

Orr, M. J. L., \& Browne, I. W. A. 1982, MNRAS, 200, 1067

Perley, R. A., Dreher, J. W., \& Cowan, J. J. 1984, ApJ, 285, L35

Perley, R. A., Röser, H.-J., \& Meisenheimer, K. 1997, A\&A, 328, 12

Röser, H.-J., \& Meisenheimer, K. 1987, ApJ, 314, 70

Rybicki, G. B., \& Lightman, A. P. 1979, Radiative Processes in Astrophysics (New York: Wiley)

Sambruna, R. M., Maraschi, L., Tavecchio, F., Urry, C. M., Cheung, C. C., Chartas, G., Scarpa, R., \& Gambill, J. K. 2002, ApJ, 571, 206

Sambruna, R. M., Urry, C. M., Tavecchio, F., Maraschi, L., Scarpa, R., Chartas, G., \& Muxlow, T. 2001, ApJ, 549, L161

Spinrad, H., Djorgovski, S., Marr, J., \& Aguilar, L. 1985, PASP, 97, 932

Tavecchio, F., Maraschi, L., Sambruna, R. M., \& Urry, C. M. 2000, ApJ, 544, L23

Tregillis, I. L., Jones, T. W., \& Ryu, D. 2001, ApJ, 557, 475

Wilson, A. S., Young, A. J., \& Shopbell, P. L. 2000, ApJ, 544, L27

. 2001, ApJ, 547, 740

Worrall, D. M., Birkinshaw, M., Hardcastle, M. J., \& Lawrence, C. R. 2001, MNRAS, 326, 1127 